\title{
A Characterization of Quasi-Perfect Equilibria
}

\author{
Nicola Gatti ${ }^{\mathrm{a}}$, Mario Gilli ${ }^{\mathrm{b}}$, Alberto Marchesi ${ }^{\mathrm{a}}$ \\ ${ }^{a}$ Dipartimento di Elettronica, Informazione e Bioningegneria, Politecnico di Milano, Piazza Leonardo da Vinci 32, \\ 20133 Milano, Italy \\ ${ }^{b}$ Department of Economics, Management, and Statistics, Università degli Studi di Milano Bicocca, Piazza \\ dell'Ateneo Nuovo 1, 20126 Milano, Italy
}

\begin{abstract}
We provide a characterization of quasi-perfect equilibria in $n$-player games, showing that any quasiperfect equilibrium can be obtained as limit point of a sequence of Nash equilibria of a certain class of perturbed games in sequence form, and any limit point of a sequence of Nash equilibria of these perturbed games is a quasi-perfect equilibrium. We prove that, in games with three or more players, we need trembles defined as rational functions of the perturbation magnitude $\varepsilon$, whereas, in two-player games with nature, trembles expressed in terms of polynomial functions of $\varepsilon$ suffice. Exploiting the relationship between sequence form and extensive form, we also provide a similar characterization in terms of perturbed games in extensive form, though not compliant with Selten's definition of perturbed game.
\end{abstract}

Key words: Quasi-perfect equilibrium, Characterization, Trembles, Games in sequence form

JEL classification: C72; C61; C63

\section{Introduction}

Perturbed games and trembles are ubiquitous in the theory of equilibrium refinements since the seminal work by Selten (1975). Motivated by the consideration that a player may be more concerned with trembles of others than with her own, van Damme (1984) introduces the concept of quasi perfection. In a quasi-perfect equilibrium, each player follows a strategy which specifies an optimal choice against trembles of the other players at each information set, without taking into account her own trembles, except to the extent that they may make one of her information sets reached that otherwise would not be. Quasi-perfect equilibria have some attractive properties. In particular, van Damme (1984) proves that a proper equilibrium of a game in normal form is a quasi-perfect equilibrium in any game in extensive form having that game as normal form. Furthermore, in a quasi-perfect equilibrium, each player plays optimal admissible continuations from every information set of her (Blume et al., 1991). Because of these properties, it has been argued by Mertens (1995) that quasi perfection is conceptually superior to normal-form perfection and extensive-form perfection (Selten, 1975). This view is shared by Govindan and Wilson (2006, 2012), who put quasi perfection at the center of their axiomatic equilibrium selection theory.

Unfortunately, while normal-form perfect equilibria and extensive-form perfect equilibria have been characterized as limit points of sequences of Nash equilibria of perturbed games in normal form and in extensive form, respectively, no similar characterization of quasi-perfect equilibria is known. The only known result is Lemma 1 by Miltersen and Sørensen (2010), showing that, in two-player games, limit points of sequences of Nash equilibria of a particular class of perturbed 
games in sequence form are quasi-perfect equilibria of the original game in extensive form. However, as the authors point out, their result only applies to a subset of the quasi-perfect equilibria in two-player games with nature. The lack of a characterization of quasi-perfect equilibria prevents the design of algorithms dealing with them. In particular, there are no viable, algorithmic techniques that perform tasks such as, e.g., verifying whether a given strategy profile is a quasiperfect equilibrium or not, enumerating all the extremal quasi-perfect equilibria, or computing a social-welfare-maximizing equilibrium.

In this paper, we provide a complete characterization of quasi-perfect equilibria in $n$-player games. We define a specific class of perturbed games in sequence form, showing that any quasiperfect equilibrium is a limit point of a sequence of Nash equilibria of perturbed games in our class and, conversely, any limit point of a sequence of Nash equilibria of our perturbed games is a quasi-perfect equilibrium. Our perturbed games satisfy two crucial properties. Firstly, the trembles are expressed as rational functions of the perturbation magnitude $\varepsilon$. Secondly, for every pair of sequences such that one directly extends the other, the tremble applied to the former approaches zero faster than that applied to the latter (as $\varepsilon$ goes to zero). In the specific case of two-player games with nature, we prove that trembles defined as polynomial functions of $\varepsilon$ suffice, while, with three or more players, they do not allow to capture the entire set of quasi-perfect equilibria and, thus, trembles defined as rational functions are necessary. Finally, exploiting the relationship between sequence form and extensive form, we also provide a similar characterization of quasi-perfect equilibria in terms of perturbed games in extensive form, though not compliant with Selten's original definition of perturbed game.

Our characterization provides new insights into the computational problem of verifying whether a given strategy profile is a quasi-perfect equilibrium. Specifically, for two-player games with nature, Gatti et al. (2016) provide a polynomial-time algorithm to verify whether a given strategy profile is a limit point of a sequence of Nash equilibria of perturbed games with trembles defined as polynomial functions of $\epsilon$. Our results show that polynomial trembles are sufficient in two-player games with nature, and, thus, in such setting the algorithm by Gatti et al. (2016) can solve the quasi-perfect equilibrium verification problem in polynomial time. Our results also show that this algorithm cannot be directly extended to games with three or more players, since trembles defined as rational functions are necessary. ${ }^{1}$

The paper is structured as follows. Section 2 recalls the notions of game in extensive and in sequence form, and revises the concept of quasi-perfect equilibrium. Section 3 introduces our class of perturbed games in sequence form and studies the properties of Nash equilibria in these perturbed games, providing the conceptual tools required by Sections 4 and 5, which are the core of the paper. Section 4 proves that any limit point of a sequence of Nash equilibria of our perturbed games is a quasi-perfect equilibrium of the original game, while Section 5 proves that any quasi-perfect equilibrium can be obtained as limit point of a sequence of Nash equilibria of perturbed games, completing the characterization. Moreover, the last part of Section 5 also shows that polynomial trembles are sufficient for our characterization in two-player games with nature. Section 6 concludes our work with two remarks. First, it shows that polynomial trembles are not sufficient with three or more players, and, then, it provides a characterization of quasi-perfect equilibria using perturbed games in extensive form. All the omitted proofs are in the Appendix.

\footnotetext{
${ }^{1}$ In the case of games with three or more players, Hansen et al. (2010) show that the verification problem of quasi-perfect equilibrium is hard.
} 


\begin{tabular}{|c|c|}
\hline Symbol & Meaning \\
\hline$T$ & game tree \\
\hline$O$ & root of the game tree \\
\hline$Z$ & terminal nodes of the game tree \\
\hline$K=X \cup Z$ & decision nodes of the game tree \\
\hline$N=\{1, \ldots, n\}$ & nodes of the game tree \\
\hline 0 & chancers player \\
\hline$\iota: X \rightarrow N \cup\{0\}$ & who plays at each decision node \\
\hline$u \in U_{i}$ & information set of player $i$ \\
\hline$C_{u}$ & set of choices at information set $u \in U$ \\
\hline$D_{i}$ & set of all choices of player $i$ \\
\hline$\beta_{i} \in B_{i}$ & player $i$ 's behavior strategy \\
\hline$\beta \in B$ & behavior strategy profile \\
\hline$h_{i}: Z \rightarrow \mathbb{R}$ & player $i$ 's payoff function \\
\hline$Z(u) \subseteq Z$ & set of leaves reachable from $u \in U$ \\
\hline$H_{i}(\beta \mid u)$ & player $i$ 's expected payoff associated to $\beta \in B$ \\
& and conditioned on $u \in U_{i}$ being reached \\
\hline$p(x)$ & choices on the path to a node $x \in K$ \\
\hline$u \preceq v$ & $u \in U_{i}$ precedes $v \in U_{i}$ \\
\hline$u \prec v$ & precedes $v \in U_{i}$ and $u \neq v$ \\
\hline
\end{tabular}

Table 1: Notation for games in extensive form.

\section{Preliminaries}

In this section, we review notation and definitions required for our results.

\subsection{Game Forms}

Our notation and conventions for games in extensive form follow Selten (1975) and von Stengel (1996), and they are summarized in Table 1. A finite game in extensive form is a tuple $\Gamma=$ $\left(T, N, \iota, U, D, \beta_{0}, h\right)$, where: $T$ is the finite game tree; $N=\{1, \ldots, n\}$ is the set of players; $\iota$ : $X \rightarrow N \cup\{0\}$ is the player function (with 0 denoting the chance player); $U=\bigcup_{i \in N \cup\{0\}} U_{i}$ is the information partition; $D=\bigcup_{i \in N \cup\{0\}} D_{i}$ is the set of possible players' choices; $\beta_{0}$ defines the fixed positive probability distributions for the chance moves; and $h=\left(h_{i}\right)_{i \in N}$ is the payoff vector function. In what follows, we assume that the game has perfect recall, and, thus, we can restrict the attention to behavior strategies. Formally, a behavior strategy $\beta_{i} \in B_{i}$ of player $i$ defines a probability distribution over the player's choices $c \in C_{u}$ at each player $i$ 's information set $u \in U_{i}$.

von Stengel (1996) defines the sequence form for any game in extensive form with perfect recall. The sequence form is a strategic description which represents strategies as sequences of a player's choices rather than as complete plans of moves, as it is the case for pure strategies in the normal form. For every player $i \in N \cup\{0\}$, any node $x \in K$ of the game tree identifies a sequence of player $i$, which is defined as the ordered list of player $i$ 's choices encountered on the path from the root of the game tree $o$ to the node $x$.

A sequence $s_{i}$ can be regarded as a string of player $i$ 's choices in $D_{i}$ found on the path to some node $x$. However, for easy reference to its elements, we can define sequence $s_{i}$ as the set 
of its choices. This is possible assuming, without loss of generality, that choices at different information sets are distinct. Notice that, for each player, there are at most as many sequences as the number of nodes of the game tree; so their number is linear in the size of the game tree. In contrast, the number of pure strategies of the normal form may be exponential in the size of the tree. The set of all sequences of player $i$ is denoted by $S_{i}$, while a sequence profile is a tuple $s=\left(s_{0}, s_{1}, \ldots, s_{n}\right) \in S_{0} \times S_{1} \times \ldots \times S_{n}=S$.

By definition of perfect recall, every node in an information set $u \in U_{i}$ defines the same sequence of choices for player $i$, hence the following definitions are well posed. Following von Stengel (1996), we denote with $\sigma_{u}$ the sequence of choices of the player acting at information set $u \in U$ that lead to $u$. For any $u \in U$ and $c \in C_{u}$, the sequence $\sigma_{u}$ can be extended by adding the choice $c$ at the end, i.e., we can write $\sigma_{u} c=\sigma_{u} \cup c$. Thus, the set $S_{i}$ of player $i$ 's sequences can be represented as $S_{i}=\{\varnothing\} \cup\left\{\sigma_{u} c \mid u \in U_{i}, c \in C_{u}\right\}$. Notice that $\sigma_{o}=\varnothing$, hence the empty sequence $\varnothing$ belongs to $S_{i}$, for any $i \in N \cup\{0\}$. In order to simplify notation, given a sequence $s_{i} \in S_{i}$ and a choice $c \in D_{i}$, we denote as $s_{i} c$ the extended sequence obtained by appending $c$ to $s_{i}$ (if the resulting sequence is well-defined for player $i$ ). For every pair of sequences $s_{i}, s_{i}^{\prime} \in S_{i}$, we write $s_{i}^{\prime} \sqsubseteq s_{i}$ whenever the set of choices defining sequence $s_{i}^{\prime}$ is a subset of that defining sequence $s_{i}$. We remark that the sequences of the chance player 0 are also considered, since it is treated as the other players.

In order to define the payoffs associated to sequence profiles, note that, for any node $x \in K$, there exists a unique sequence profile $s=\left(s_{0}, s_{1}, \ldots, s_{n}\right) \in S$ leading to $x$. Hence, we can define the function $\xi: K \rightarrow S$ specifying the sequence profile reaching $x \in K$. Then, according to von Stengel (1996), in a game in sequence form, the player $i$ 's payoff function is $g_{i}: S \rightarrow \mathbb{R}$, where:

$$
g_{i}(s)= \begin{cases}h_{i}(z) & \text { if } \exists z \in Z \text { s.t. } \quad s=\left(s_{0}, s_{1}, \ldots, s_{n}\right)=\xi(z) \\ 0 & \text { otherwise }\end{cases}
$$

The payoff function $g_{i}$ is commonly represented as a sparse $(n+1)$-dimensional matrix, where the number of nonzero entries is at most the number of leaves of the game tree.

In addition to the payoffs, it is also necessary to specify how sequences are selected by a player. Behavior strategy probabilities will be replaced by the realization probabilities of sequences. In games in sequence form, a player cannot just decide on a single sequence. For any player $i$, the realization plan of a behavior strategy $\beta_{i} \in B_{i}$ is the function $r_{i}: S_{i} \rightarrow \mathbb{R}^{+}$defined as follows: for any sequence $s_{i} \in S_{i}$, it holds $r_{i}\left(s_{i}\right)=\prod_{c \in s_{i}} \beta_{i}(c)$. Then, for any information set $u \in U_{i}$ and choice $c^{\prime} \in C_{u}$, we have $r_{i}\left(\sigma_{u} c^{\prime}\right)=\prod_{c \in \sigma_{u}} \beta_{i}(c) \beta_{i}\left(c^{\prime}\right)$. By construction, a realization plan of a behavior strategy is characterized by the following linear restrictions:

$$
r_{i}(\varnothing)=1
$$

because the empty product is 1 ; for any information set $u \in U_{i}$

$$
-r_{i}\left(\sigma_{u}\right)+\sum_{c \in C_{u}} r_{i}\left(\sigma_{u} c\right)=0
$$

because $\sum_{c \in C_{u}} \beta_{i}(c)=1$; and for every sequence $s_{i} \in S_{i}$

$$
r_{i}\left(s_{i}\right) \geq 0
$$

because it is a probability. This leads to the following definition: a function $r_{i}: S_{i} \rightarrow \mathbb{R}^{+}$fulfilling restrictions (1), (2), and (3) is a realization plan for player $i \in N$. Moreover, notice that the 


\begin{tabular}{|c|c|}
\hline Symbols & Meaning \\
\hline$s_{i} \in S_{i}$ & sequence of player $i$ \\
\hline$s \in S$ & sequence profile \\
\hline$\sigma_{u}$ & sequence of the player acting at $u \in U$ that leads to $u$ \\
\hline$\sigma_{u} c$ & sequence leading to $u \in U$ extended by $c \in C_{u}$ \\
\hline$\xi: K \rightarrow S$ & function defining the sequence profile reaching $x \in K$ \\
\hline$g_{i}: S \rightarrow \mathbb{R}$ & player $i$ 's payoff function in the sequence form \\
\hline$r_{i}: S_{i} \rightarrow \mathbb{R}^{+}$ & player $i$ 's realization plan \\
\hline$r / r_{i}^{\prime}$ & realization plan profile obtained from $r \in R$ \\
by replacing $r_{i}$ with $r_{i}^{\prime} \in R_{i}$
\end{tabular}

Table 2: Notation for games in sequence form.

chance player has a fixed realization plan $r_{0}$ obtained from the behavior strategy $\beta_{0}$. The set of all realization plans for player $i \in N$ is denoted by $R_{i}$, while, for notational convenience, we let $R_{0}=\left\{r_{0}\right\}$. Then, $r=\left(r_{0}, r_{1}, \ldots, r_{n}\right) \in R_{0} \times R_{1} \times \ldots \times R_{n}=R$ is a tuple representing a realization plan profile. We also use $r / r_{i}^{\prime}$ to denote the realization plan profile obtained from $r \in R$ by replacing $r_{i}$ with $r_{i}^{\prime} \in R_{i}$ for some player $i \in N$.

Let us recall that von Stengel (1996) shows that any realization plan $r_{i}: S_{i} \rightarrow \mathbb{R}^{+}$satisfying linear restrictions (1), (2), and (3) defines a suitable behavior strategy such that $\beta_{i}(c)=\frac{r_{i}\left(\sigma_{u} c\right)}{r_{i}\left(\sigma_{u}\right)}$ for all $u \in U_{i}$ and $c \in C_{u}$ if $r_{i}\left(\sigma_{u}\right)>0$, while $\beta_{i}(c)$ can be any otherwise.

The game in sequence form associated to a finite game in extensive form with perfect recall $\Gamma$ is the tuple $\Gamma_{S}=\left(S, N, R, r_{0}, g\right)$, where: $S=S_{0} \times S_{1} \times \ldots \times S_{n}$ is the set of sequence profiles; $N=\{1, \ldots, n\}$ is the set of players; $R=R_{0} \times R_{1} \times \ldots \times R_{n}$ is the set of realization plan profiles; $r_{0}$ is the realization plan of $\beta_{0}$; and $g=\left(g_{i}\right)_{i \in N}$ is the payoff vector function of the sequence form. As for games in extensive form, for any player $i \in N$, we define the player $i$ 's expected payoff associated to a realization plan profile $r \in R$ as:

$$
G_{i}(r)=\sum_{s \in S} g_{i}(s) \prod_{j \in N \cup\{0\}} r_{j}\left(s_{j}\right) .
$$

Table 2 sums up the notation used for games in sequence form.

\subsection{Quasi-Perfect Equilibria}

Given two player $i$ 's behavior strategies $\beta_{i}, \beta_{i}^{\prime} \in B_{i}$ and an information set $u \in U_{i}$, we write $\beta_{i}=\beta_{i}^{\prime}$ whenever $\beta_{i}$ and $\beta_{i}^{\prime}$ prescribe the same probability distribution over the choices available at information set $u$, i.e., $\beta_{i}(c)=\beta_{i}^{\prime}(c)$ for every $c \in C_{u}$. Furthermore, given $\beta_{i}, \beta_{i}^{\prime} \in B_{i}$ and $u \in U_{i}$, we write $\beta_{i} /{ }_{u} \beta_{i}^{\prime}$ to denote a behavior strategy which is equal to $\beta_{i}^{\prime}$ at all information sets $v \in U_{i}$ such that $u \preceq v$, while it is equal to $\beta_{i}$ at the other information sets (see Table 1 for the definition of the relation $\preceq$ ). In addition, overloading notation, given $\beta \in B, \beta_{i}^{\prime} \in B_{i}$, and $u \in U_{i}, \beta /{ }_{u} \beta_{i}^{\prime}$ denotes a strategy profile obtained from $\beta$ by replacing player $i$ 's strategy $\beta_{i}$ with $\beta_{i} /{ }_{u} \beta_{i}^{\prime}$. 
Definition 1. A family of parameterized behavior strategy profiles, denoted by $\mathcal{P}$, is a family of functions $\boldsymbol{\beta} \in \mathcal{P}$ such that $\boldsymbol{\beta}:(0,1) \rightarrow B$ maps any number $\varepsilon \in(0,1)$ to a behavior strategy profile $\boldsymbol{\beta}(\varepsilon) \in B$. Moreover:

- $\boldsymbol{\beta} \in \mathcal{P}$ is completely mixed if $\boldsymbol{\beta}(\varepsilon)$ is always completely mixed;

- $\boldsymbol{\beta} \in \mathcal{P}$ is rational (respectively polynomial) if each probability $\boldsymbol{\beta}_{i}(\varepsilon, c)$ can be expressed as a rational (respectively polynomial) function of $\varepsilon$.

Definition 2. Given a game in extensive form $\Gamma$, a completely mixed behavior strategy profile $\beta \in B$, a player $i \in N$, and an information set $u \in U_{i}$, a behavior strategy $\beta_{i} \in B_{i}$ is a $u$-best response to $\beta \in B$, written $\beta_{i} \in \mathcal{B R}_{u}(\beta)$, if the following holds:

$$
\max _{\beta_{i}^{\prime} \in B_{i}: \beta_{i}^{\prime}=\beta_{i}} H_{i}\left(\beta /{ }_{u} \beta_{i}^{\prime} \mid u\right)=\max _{\beta_{i}^{\prime} \in B_{i}} H_{i}\left(\beta /{ }_{u} \beta_{i}^{\prime} \mid u\right) .
$$

Intuitively, $\beta_{i}$ is a $u$-best response to $\beta$ whenever playing as prescribed by $\beta_{i}$ at information set $u$ is part of some player $i$ 's optimal strategy in the game following $u$, given that $u$ has been reached and the other players behave as prescribed by $\beta$. In other words, $\beta_{i}$ is a best response to $\beta$ whenever player $i$ ends up playing at information set $u .^{2}$

A formal definition of quasi-perfect equilibrium is provided by van Damme (1984), as follows. ${ }^{3}$

Definition 3. Given a game in extensive form $\Gamma, \beta \in B$ is a quasi-perfect equilibrium of $\Gamma$ if there exists a completely mixed $\boldsymbol{\beta} \in \mathcal{P}$ such that:

1. $\beta$ is a limit point (as $\varepsilon$ goes to zero) of $\boldsymbol{\beta}(\varepsilon)$;

2. for every player $i \in N$ and information set $u \in U_{i}, \beta_{i} \in \mathcal{B R}_{u}(\boldsymbol{\beta}(\varepsilon))$ holds eventually (i.e., for sufficiently small $\varepsilon$ ).

In words, provided that $\varepsilon \in(0,1)$ is sufficiently small, for every player $i$ and information set $u \in U_{i}, \beta_{i}$ prescribes player $i$ to play an optimal strategy against $\boldsymbol{\beta}(\varepsilon)$, given that $u$ has been reached and she will play optimally in the rest of the game (assuming opponents follow strategies in $\boldsymbol{\beta}(\varepsilon)$ ). This condition encodes the fact that a player only considers the possibility of opponents' future trembles, while she does not care about hers.

Notice that, in order to span the entire set of quasi-perfect equilibria, it is necessary to use nonpolynomial $\boldsymbol{\beta} \in \mathcal{P}$. In particular, rational restrictions are required any time in an equilibrium we have to combine sequential rationality and completely mixed behavior strategies. This is formally shown by Theorem 4.1 of Hammond (1994), where he proves that equilibrium refinements have four equivalent characterizations: (i) complete conditional probability systems; ${ }^{4}$ (ii) lexicographic hierarchies of probabilities; ${ }^{5}$ (iii) extended logarithmic likelihood ratios; and (iv) certain "canonical rational probability functions" representing "trembles" directly as infinitesimal probabilities. In particular, Kohlberg and Reny (1997) prove that sequential equilibria can be characterized in terms of relative probabilities that compare the likelihoods of any pair of events, even those with probability zero. ${ }^{6}$ More generally, Blume and Zame (1994) prove that the equilibrium correspondences of extensive-form perfect and sequential equilibria are semi-algebraic sets and, therefore,

\footnotetext{
${ }^{2}$ In the terminology of van Damme (1984), $u$-best responses are quasi-best replies.

${ }^{3}$ See also van Damme (1984) Lemma 2 and Govindan and Wilson (2012) Definition 4.1.

${ }^{4}$ See Myerson (1986) and McLennan (1989).

${ }^{5}$ See Blume et al. (1991) and Govindan and Klumpp (2003).

${ }^{6}$ Note that quasi-perfect equilibria are a refinement of sequential equilibria.
} 
they can be defined by finite systems of polynomial inequalities. Finally, Hillas et al. (2017) and Pimienta and Shen (2014) extended Blume and Zame (1994) proving that also the equilibrium correspondences of quasi-perfect equilibria are semi-algebraic. For the sake of this paper, we can sum up these considerations with the following remark.

Remark 1. Given a game in extensive form $\Gamma$, let $\beta \in B$ be a quasi-perfect equilibrium of $\Gamma$. Then, there exists a completely mixed $\boldsymbol{\beta} \in \mathcal{P}$ such that $\boldsymbol{\beta}$ is rational and conditions (1) and (2) in Definition 3 hold.

\section{Nash Equilibria of Perturbed Games}

In this section, we introduce our class of $n$-player perturbed games in sequence form and prove some properties of their Nash equilibria, which turn out to be crucial in proving our core results in the following Sections 4 and 5.

\subsection{A General Approach to Perturbation in Sequence Form}

The following definition formally introduces our perturbations.

Definition 4. Given a game in sequence form $\Gamma_{S}$ and a player $i \in N$, let $\ell_{i}:(0,1) \times S_{i} \mapsto \mathbb{R}_{>0}{ }^{7}$ be a function that maps any number $\varepsilon \in(0,1)$ and sequence $s_{i} \in S_{i}$ to a lower bound $\ell_{i}\left(\varepsilon, s_{i}\right)$ such that:

1. $\lim _{\varepsilon \rightarrow 0^{+}} \ell_{i}\left(\varepsilon, s_{i}\right)=0$, for every $s_{i} \in S_{i} \backslash\{\varnothing\}$, while $\ell_{i}(\varepsilon, \varnothing)=1$;

2. $\lim _{\varepsilon \rightarrow 0^{+}} \frac{\ell_{i}\left(\varepsilon, s_{i} c\right)}{\ell_{i}\left(\varepsilon, s_{i}\right)}=0$, for every $s_{i} \in S_{i}$ and $c \in D_{i}$ such that $s_{i} c \in S_{i}$.

Then, we define an $\ell_{i}$-quasi-perfect perturbation for $R_{i}$ as a function $\varepsilon \mapsto R_{i}(\varepsilon)$ defined over $\varepsilon \in(0,1)$ in which $R_{i}(\varepsilon)$ is the set of all the realization plans $r_{i} \in R_{i}$ such that $r_{i}\left(s_{i}\right) \geq \ell_{i}\left(\varepsilon, s_{i}\right)$ for every $s_{i} \in S_{i}$.

In words, an $\ell_{i}$-quasi-perfect perturbation constrains player $i$ to play every sequence $s_{i} \in S_{i}$ with probability larger than or equal to the lower bound (or tremble) $\ell_{i}\left(\varepsilon, s_{i}\right)$. Furthermore, the lower bounds enjoy particular properties:

1. they approach zero as $\varepsilon$ goes to zero;

2. for every sequence $s_{i} \in S_{i}$ and choice $c \in D_{i}$ such that $s_{i} c \in S_{i}, \ell_{i}\left(\varepsilon, s_{i} c\right)$ approaches zero faster than $\ell_{i}\left(\varepsilon, s_{i}\right)$.

Definition 5. Given a game in extensive form $\Gamma$, we call perturbed sequence form of $\Gamma$ the sequence form $\Gamma_{S}$ associated with $\Gamma$ together with an $\ell_{i}$-quasi-perfect perturbation for $R_{i}$, for every player $i \in N$.

In the following, the tuple $\left(\Gamma_{\boldsymbol{S}},\left\{\ell_{i}\right\}_{i \in N}\right)$ denotes a perturbed sequence form of $\Gamma$, defined for some $\ell_{i}$-quasi-perfect perturbations. Moreover, we let $\Gamma_{S}(\varepsilon)$ be a particular perturbed game in sequence form, which is obtained from $\Gamma_{S}$ by restricting each set of realization plans $R_{i}$ to be $R_{i}(\varepsilon){ }^{8}$ In the following, we use $r(\varepsilon)$ to denote a valid realization plan profile for a perturbed game in sequence form $\Gamma_{\boldsymbol{S}}(\varepsilon)$, i.e., $r_{i}(\varepsilon) \in R_{i}(\varepsilon)$ for all $i \in N$.

\footnotetext{
${ }^{7}$ We denote with $\mathbb{R}_{>0}$ the set of strictly positive real numbers.

${ }^{8}$ Let us remark that $\Gamma_{\boldsymbol{S}}(\varepsilon)$ may not be well-defined for all values of $\varepsilon \in(0,1)$, since restrictions $(2)$ and $(3)$ may not be satisfied. Nevertheless, conditions (1) and (2) in Definition 4 ensure that there exists $\bar{\varepsilon} \in(0,1)$ such that $\Gamma_{\boldsymbol{S}}(\varepsilon)$ is well-defined for all $\varepsilon \in(0, \bar{\varepsilon})$. Thus, whenever we refer to a perturbed game in sequence form $\Gamma_{S}(\varepsilon)$, we can safely assume that it is well-defined, provided that $\varepsilon$ is sufficiently small.
} 


\subsection{Properties of the Nash Equilibria of Perturbed Games}

The main result of this section (Theorem 1) provides a characterization of the Nash equilibria of our perturbed games in sequence form, which is a fundamental step for our characterization of quasi-perfect equilibria. We report its complete proof in the Appendix. Intuitively, it leverages the interplay between the properties of the mathematical programming problem for finding a Nash equilibrium in games in sequence form and those of our perturbed games.

For the ease of presentation, given a player $i \in N$ and an information set $u \in U_{i}$, we let

$$
G_{i}(u, r)=\sum_{c \in C_{u}} \sum_{s \in S: \sigma_{u} c \sqsubseteq s_{i}} g_{i}(s) \prod_{j \in N \cup\{0\}} r_{j}\left(s_{j}\right)
$$

be player $i$ 's expected utility contribution due to terminal nodes reachable from $u$, given that the realization plan profile $r \in R$ is played.

Theorem 1. Given a perturbed sequence form $\left(\Gamma_{\boldsymbol{S}},\left\{\ell_{i}\right\}_{i \in N}\right)$, a realization plan profile $r(\varepsilon)$ is a Nash equilibrium of $\Gamma_{\boldsymbol{S}}(\varepsilon)$ if and only if, for every player $i \in N$, information set $u \in U_{i}$, and choice $c \in C_{u}$, it holds that:

$$
r_{i}\left(\varepsilon, \sigma_{u} c\right)>\ell_{i}\left(\varepsilon, \sigma_{u} c\right) \Longrightarrow \max _{\substack{r_{i}^{\prime} \in R_{i}: \\ r_{i}^{\prime}\left(\sigma_{u} c\right)=1}} G_{i}\left(u, r(\varepsilon) / r_{i}^{\prime}\right)=\max _{\substack{r_{i}^{\prime} \in R_{i}: \\ r_{i}^{\prime}\left(\sigma_{u}\right)=1}} G_{i}\left(u, r(\varepsilon) / r_{i}^{\prime}\right) .
$$

In words, in a Nash equilibrium of $\Gamma_{\boldsymbol{S}}(\varepsilon)$, player $i$ plays sequence $\sigma_{u} c$ with probability strictly greater than its lower bound $\ell_{i}\left(\varepsilon, \sigma_{u} c\right)$ only if playing $c$ is part of some optimal strategy in the game following $u$. Conversely, whenever $c \in C_{u}$ is not part of any optimal strategy in the game following $u$, it must be the case that sequence $\sigma_{u} c$ is played with probability equal to its lower bound. Theorem 1 formally expresses the idea that, in a perturbed game in sequence form $\Gamma_{\boldsymbol{S}}(\varepsilon)$, when a player decides how to play in a given information set, she does not take into account her future trembles, but only opponents' ones.

\section{Nash Equilibria of Perturbed Games in Sequence Form are Quasi-Perfect Equilibria}

In this section, we prove the first part of our main result (Theorem 2), i.e., all the limit points of sequences of Nash equilibria of our perturbed games $\Gamma_{\boldsymbol{S}}(\varepsilon)$ are quasi-perfect equilibria of the game in extensive form $\Gamma$ (as $\varepsilon$ goes to zero).

Definition 6. A family of parameterized realization plan profiles, denoted by $\mathcal{R}$, is a family of functions $\boldsymbol{r} \in \mathcal{R}$ such that $\boldsymbol{r}:(0,1) \mapsto R$ maps any number $\varepsilon \in(0,1)$ to a realization plan profile $\boldsymbol{r}(\varepsilon) \in R$. Moreover:

- $\boldsymbol{r} \in \mathcal{R}$ is completely mixed if $\boldsymbol{r}(\varepsilon)$ is always completely mixed;

- $\boldsymbol{r} \in \mathcal{R}$ is rational (respectively polynomial) if each term $\boldsymbol{r}_{i}\left(\varepsilon, s_{i}\right)$ can be expressed as a rational (respectively polynomial) function of $\varepsilon$.

The following lemma (whose proof is in the Appendix) provides a characterization of $u$-best responses in terms of the sequence form. 
Lemma 1. Given a game in extensive form $\Gamma$, a completely mixed behavior strategy profile $\beta \in B$, a player $i \in N$, and an information set $u \in U_{i}$, a behavior strategy $\beta_{i} \in B_{i}$ is a u-best response to $\beta \in B$, i.e., $\beta_{i} \in \mathcal{B R}_{u}(\beta)$, if and only if the following holds for every choice $c \in C_{u}$ :

$$
\beta_{i}(c)>0 \Longrightarrow \max _{\substack{r_{i}^{\prime} \in R_{i}: \\ r_{i}^{\prime}\left(\sigma_{u} c\right)=1}} G_{i}\left(u, r / r_{i}^{\prime}\right)=\max _{\substack{r_{i}^{\prime} \in R_{i}: \\ r_{i}^{\prime}\left(\sigma_{u}\right)=1}} G_{i}\left(u, r / r_{i}^{\prime}\right),
$$

where $r \in R$ is the realization plan profile of $\beta$.

Intuitively, a behavior strategy $\beta_{i}$ is a $u$-best response to $\beta$ if and only if it places positive probability only on choices $c \in C_{u}$ which are part of some player $i$ 's optimal strategy in the game following information set $u$.

Theorem 2. Given a perturbed sequence form $\left(\Gamma_{\boldsymbol{S}},\left\{\ell_{i}\right\}_{i \in N}\right)$, let:

1. $\boldsymbol{r} \in \mathcal{R}$ be such that $\boldsymbol{r}(\varepsilon)$ is a Nash equilibrium of $\Gamma_{\boldsymbol{S}}(\varepsilon)$;

2. $\boldsymbol{\beta} \in \mathcal{P}$ be such that $\boldsymbol{\beta}(\varepsilon)$ has $\boldsymbol{r}(\varepsilon)$ as realization plan profile.

Then, any limit point (as $\varepsilon$ goes to zero) of $\boldsymbol{\beta}(\varepsilon)$ is a quasi-perfect equilibrium of $\Gamma$.

Proof. Let us fix $\boldsymbol{r} \in \mathcal{R}$ such that $\boldsymbol{r}(\varepsilon)$ is a Nash equilibrium of $\Gamma_{\boldsymbol{S}}(\varepsilon)$, and let $\beta \in B$ be a limit point (as $\varepsilon$ goes to zero) of $\boldsymbol{\beta}(\varepsilon)$. In order to prove that $\beta$ is a quasi-perfect equilibrium of $\Gamma$, it is sufficient to show that condition (2) in Definition 3 holds. First, notice that there must exist $\bar{\varepsilon} \in(0,1)$ such that, for all $\varepsilon \in(0, \bar{\varepsilon})$, and for every player $i \in N$, information set $u \in U_{i}$, and choice $c \in \sigma_{u}$, if $\beta_{i}(c)>0$, then $\boldsymbol{r}_{i}\left(\varepsilon, \sigma_{u} c\right)>\ell_{i}\left(\varepsilon, \sigma_{u} c\right)$. Otherwise, by conditions (1) and (2) in Definition 4, it would be $\beta_{i}(c)=0$. Let us fix $i \in N, u \in U_{i}$, and $c \in \sigma_{u}$. Suppose that $\beta_{i}(c)>0$. For all $\varepsilon \in(0, \bar{\varepsilon})$, we have that $\boldsymbol{r}_{i}\left(\varepsilon, \sigma_{u} c\right)>\ell_{i}\left(\varepsilon, \sigma_{u} c\right)$, which, by Theorem 1, implies the following:

$$
\max _{\substack{r_{i}^{\prime} \in R_{i} \\ r_{i}^{\prime}\left(\sigma_{u} c\right)=1}} G_{i}\left(u, \boldsymbol{r}(\varepsilon) / r_{i}^{\prime}\right)=\max _{\substack{r_{i}^{\prime} \in R_{i}: \\ r_{i}^{\prime}\left(\sigma_{u}\right)=1}} G_{i}\left(u, \boldsymbol{r}(\varepsilon) / r_{i}^{\prime}\right) .
$$

Thus, Lemma 1 allows us to conclude that $\beta_{i} \in \mathcal{B R}_{u}(\boldsymbol{\beta}(\varepsilon))$ eventually, i.e., for $\varepsilon \in(0, \bar{\varepsilon})$. This implies that $\beta \in B$ is a quasi-perfect equilibrium of $\Gamma$.

\subsection{Discussion on Miltersen-Sørensen Perturbed Games}

Miltersen and Sørensen (2010) study a particular class of perturbed games in sequence form in which the lower bounds on the probabilities of playing sequences $s_{i} \in S_{i}$ are defined as $\varepsilon^{\left|s_{i}\right|}$, where $\left|s_{i}\right|$ denotes the number of choices in the sequence $s_{i}$ (i.e., its length). These perturbed games are a special case of ours, obtained by letting $\ell_{i}\left(\varepsilon, s_{i}\right)=\varepsilon^{\left|s_{i}\right|}$ for every player $i \in N$ and sequence $s_{i} \in S_{i}$. Clearly, lower bounds $\ell_{i}\left(\varepsilon, s_{i}\right)$ satisfy conditions (1) and (2) in Definition 4.

Miltersen and Sørensen (2010) prove that, in two-player games in extensive form with nature, any limit point of a sequence of Nash equilibria of their perturbed games is a quasi-perfect equilibrium of the original game. Thus, our Theorem 2 is a generalization of their result that works for a broader class of perturbed games in sequence form (see Definition 4) and is not restricted to the specific setting of two-player games. ${ }^{9}$ Moreover, as the authors point out, Miltersen-Sørensen

\footnotetext{
${ }^{9}$ Notice that the proof of Theorem 2 is not a generalization of the proof of Lemma 1 by Miltersen and Sørensen (2010), as it requires completely different techniques that rely on mathematical programming. We also remark that Miltersen and Sørensen (2010) restrict their attention to two-player games for computational purposes.
} 


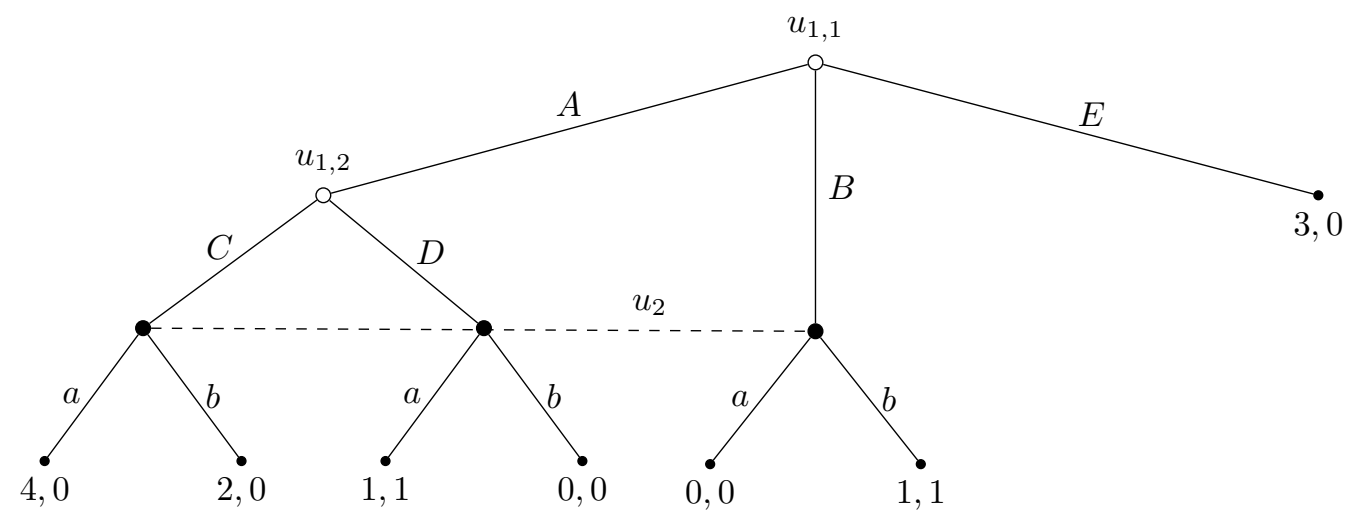

Figure 1: Example showing that Miltersen-Sørensen perturbed games do not allow to capture all the quasi-perfect equilibria, even in the basic setting of two-player games.

perturbed games do not allow to capture all the quasi-perfect equilibria, and, thus, they are not sufficient to provide a characterization of quasi-perfect equilibria (even in the two-player setting).

In Figure 1, we provide a two-player game in extensive form in which the perturbed games defined by Miltersen and Sørensen do not identify all the quasi-perfect equilibria, thus motivating the introduction of our general class of perturbed games.

In particular, in the game of Figure 1, the sets of Nash equilibria, of normal-form perfect equilibria, of extensive-form perfect equilibria, and of quasi-perfect equilibria coincide and they are the union of $\left\{\beta_{1} \in B_{1}: \beta_{1}(A)=\beta_{1}(C)=1\right\} \times\left\{\beta_{2} \in B_{2}: \beta_{2}(a) \geq \frac{1}{2}\right\}$ and $\left\{\beta_{1} \in B_{1}: \beta_{1}(E)=1\right.$, $\left.\beta_{1}(C) \in[0,1]\right\} \times\left\{\beta_{2} \in B_{2}: \beta_{2}(a) \leq \frac{1}{2}\right\}$. However, notice that, in the Miltersen-Sørensen perturbed game, it holds $r_{1}(B) \geq \varepsilon$ and $r_{2}(A D) \geq \varepsilon^{2}$, which implies that, in any Nash equilibrium of the Miltersen-Sørensen perturbed game, sequence $b$ is played with probability $1-\varepsilon$ by the second player. Then, the first player chooses sequence $E$ with probability $1-2 \varepsilon$ and sequence $A C$ with probability $\varepsilon-\varepsilon^{2}$. Hence, the Nash equilibria of the Miltersen-Sørensen perturbed game are:

$$
\left\{\beta_{1} \in B_{1}: \beta_{1}(E)=1-2 \varepsilon, \beta_{1}(C)=\frac{\varepsilon-\varepsilon^{2}}{\varepsilon}\right\} \times\left\{\beta_{2} \in B_{2}: \beta_{2}(b)=1-\varepsilon\right\} .
$$

This shows that the game in Figure 1 admits a continuum of quasi-perfect equilibria which are not limit points (as $\varepsilon$ goes to zero) of Nash equilibria of the Miltersen-Sørensen perturbed game, for instance all those with $\beta_{2}(a) \geq \frac{1}{2}$ and $\beta_{1}(A)=\beta_{1}(C)=1$. In particular, when using the Miltersen-Sørensen perturbations, there is no sequence of Nash equilibria having as limit point a quasi-perfect equilibrium in which the terminal node with payoffs $(4,0)$ is reached. Instead, using our perturbed games, it is sufficient to define $\ell_{1}(\varepsilon, A)=\ell_{1}(\varepsilon, E)=\varepsilon$ and $\ell_{1}(\varepsilon, B)=\ell_{1}(\varepsilon, A C)=$ $\ell_{1}(\varepsilon, A D)=\varepsilon^{2}$ (satisfying conditions (1) and (2) in Definition 4) to obtain as limit point of sequences of Nash equilibria all the quasi-perfect equilibria in $\left\{\beta_{1} \in B_{1}: \beta_{1}(A)=\beta_{1}(C)=1\right\} \times$ $\left\{\beta_{2} \in B_{2}: \beta_{2}(a) \geq \frac{1}{2}\right\}$.

\section{Quasi-Perfect Equilibria are Nash Equilibria of Perturbed Games in Sequence Form}

In this section, we prove the second part of our main result (Theorem 3), i.e., any quasi-perfect equilibrium of a game in extensive form $\Gamma$ can be obtained as limit point of a sequence of Nash equilibria of perturbed games in sequence form $\Gamma_{\boldsymbol{S}}(\varepsilon)$, for a suitably defined perturbed sequence form of $\Gamma$. The general idea of the proof is the following: 
1. From Remark 1, we know that any quasi-perfect equilibrium $\beta \in B$ of a game in extensive form $\Gamma$ can be obtained for some completely mixed $\boldsymbol{\beta} \in \mathcal{P}$ such that $\boldsymbol{\beta}$ is rational.

2. Letting $\boldsymbol{r} \in \mathcal{R}$ be such that $\boldsymbol{r}(\varepsilon)$ is the realization plan of $\boldsymbol{\beta}(\varepsilon)$, we define a rational $\tilde{\boldsymbol{r}} \in \mathcal{R}$ such that $\tilde{\boldsymbol{r}}(\varepsilon)=\boldsymbol{r}\left(\varepsilon^{\eta}\right)$, where $\eta=\max _{i \in N, s_{i} \in S_{i}}\left|s_{i}\right|$ is the length of the longest sequence in the game. Then, we show that:

- for every player $i \in N$ and information set $u \in U_{i}$, player $i$ 's optimal strategies in the game following information set $u$ are preserved when replacing the realization plan profile $\boldsymbol{r}(\varepsilon)$ with $\tilde{\boldsymbol{r}}(\varepsilon)$ (see Lemma 2);

- $\beta$ is a limit point (as $\varepsilon$ goes to zero) of $\tilde{\boldsymbol{\beta}}(\varepsilon)$, where $\tilde{\boldsymbol{\beta}} \in \mathcal{P}$ is such that $\tilde{\boldsymbol{r}}(\varepsilon)$ is the realization plan of $\tilde{\boldsymbol{\beta}}(\varepsilon)$;

- given our choice of $\eta$, we can build a particular perturbed sequence form $\left(\Gamma_{\boldsymbol{S}},\left\{\ell_{i}^{\beta}\right\}_{i \in N}\right)$ of $\Gamma$, which depends on the quasi-perfect equilibrium $\beta$, such that each $\tilde{\boldsymbol{r}}(\varepsilon)$ is a valid realization plan for the perturbed game $\Gamma_{S}(\varepsilon)$ (see Definition 7 ).

3. Finally, we prove that $\tilde{\boldsymbol{r}}(\varepsilon)$ defines a sequence of Nash equilibria of perturbed games $\Gamma_{\boldsymbol{S}}(\varepsilon)$.

Before showing the details of our results, notice that, given a rational $\boldsymbol{r} \in \mathcal{R}$, for every player $i \in N$ and sequence $s_{i} \in S_{i}$, we can write:

$$
\boldsymbol{r}_{i}\left(\varepsilon, s_{i}\right)=a_{0}\left(s_{i}\right)+\frac{a_{1}\left(s_{i}\right) \varepsilon+\ldots+a_{d_{N}\left(s_{i}\right)}\left(s_{i}\right) \varepsilon^{d_{N}\left(s_{i}\right)}}{1+b_{1}\left(s_{i}\right) \varepsilon+\ldots+b_{d_{D}\left(s_{i}\right)}\left(s_{i}\right) \varepsilon^{d_{D}\left(s_{i}\right)}}=a_{0}\left(s_{i}\right)+\frac{\operatorname{Poly}_{i}^{N}\left(\varepsilon, s_{i}\right)}{\operatorname{Poly}_{i}^{D}\left(\varepsilon, s_{i}\right)},
$$

where $a_{0}\left(s_{i}\right) \in[0,1], a_{j}\left(s_{i}\right) \in \mathbb{R}$ for all $j \in\left\{1, \ldots, d_{N}\left(s_{i}\right)\right\}$, and $b_{j}\left(s_{i}\right) \in \mathbb{R}$ for all $j \in\left\{1, \ldots, d_{D}\left(s_{i}\right)\right\}$ are the coefficients of the polynomials $\operatorname{Poly}_{i}^{N}\left(\varepsilon, s_{i}\right)$ and $\operatorname{Poly}_{i}^{D}\left(\varepsilon, s_{i}\right)$, whereas $d_{N}\left(s_{i}\right), d_{D}\left(s_{i}\right) \in \mathbb{N}$ denote their degrees. Moreover, given a rational $\boldsymbol{\beta} \in \mathcal{P}$, it is easy to prove that $\boldsymbol{r} \in \mathcal{R}$, where $\boldsymbol{r}(\varepsilon)$ is the realization plan of $\boldsymbol{\beta}(\varepsilon)$, is rational too. This is a direct consequence of the fact that the product of rational functions is rational.

Now, we are ready to prove our first result, i.e., for any player $i \in N$ and information set $u \in U_{i}$, replacing $\boldsymbol{r}(\varepsilon)$ with $\tilde{\boldsymbol{r}}(\varepsilon)$ does not affect player $i$ 's optimal strategies in the game following $u$, provided that $\varepsilon$ is sufficiently small. This is the fundamental step that allows us to prove that $\tilde{\boldsymbol{r}}(\varepsilon)$ defines a sequence of Nash equilibria of perturbed games (see Theorem 3). The proof of the following lemma is reported in the Appendix.

Lemma 2. Given a game in sequence form $\Gamma_{\boldsymbol{S}}$ and a rational $\boldsymbol{r} \in \mathcal{R}$, there exists $\bar{\varepsilon} \in(0,1)$ such that, for all $\varepsilon \in(0, \bar{\varepsilon})$, and for any player $i \in N$, information set $u \in U_{i}$, and choice $c \in C_{u}$, it holds:

$$
\begin{gathered}
\max _{\substack{r_{i}^{\prime} \in R_{i}: \\
r_{i}^{\prime}\left(\sigma_{u} c\right)=1}} G_{i}\left(u, \boldsymbol{r}(\varepsilon) / r_{i}^{\prime}\right)=\max _{\substack{r_{i}^{\prime} \in R_{i}: \\
r_{i}^{\prime}\left(\sigma_{u}\right)=1}} G_{i}\left(u, \boldsymbol{r}(\varepsilon) / r_{i}^{\prime}\right) \\
\max _{\substack{r_{i}^{\prime} \in R_{i}: \\
r_{i}^{\prime}\left(\sigma_{u} c\right)=1}} G_{i}\left(u, \tilde{\boldsymbol{r}}(\varepsilon) / r_{i}^{\prime}\right)=\max _{\substack{r_{i}^{\prime} \in R_{i}: \\
r_{i}^{\prime}\left(\sigma_{u}\right)=1}} G_{i}\left(u, \tilde{\boldsymbol{r}}(\varepsilon) / r_{i}^{\prime}\right),
\end{gathered}
$$

where $\tilde{\boldsymbol{r}} \in \mathcal{R}$ is such that $\tilde{\boldsymbol{r}}(\varepsilon)=\boldsymbol{r}\left(\varepsilon^{\eta}\right)$, with $\eta=\max _{i \in N, s_{i} \in S_{i}}\left|s_{i}\right| .{ }^{10}$

\footnotetext{
${ }^{10}$ The choice of $\eta$ is made in such a way that it is always possible to built a perturbed sequence form $\left(\Gamma_{\boldsymbol{S}},\left\{\ell_{i}^{\beta}\right\}_{i \in N}\right)$ (see Definition 7). Notice that the lemma is still valid if we use any $\eta \in \mathbb{N}$, while we remark that the following Theorem 3 may not hold if $\eta$ is arbitrary.
} 
Notice that, letting $\tilde{\boldsymbol{\beta}} \in \mathcal{P}$ be such that $\tilde{\boldsymbol{r}}(\varepsilon)$ is the realization plan of $\tilde{\boldsymbol{\beta}}(\varepsilon)$, it is a direct consequence of the definition of $\tilde{\boldsymbol{r}}$ that $\boldsymbol{\beta}$ and $\tilde{\boldsymbol{\beta}}$ have the same limit points (as $\varepsilon$ goes to zero), i.e.,

$$
\lim _{\varepsilon \rightarrow 0^{+}} \boldsymbol{\beta}(\varepsilon)=\lim _{\varepsilon \rightarrow 0^{+}} \tilde{\boldsymbol{\beta}}(\varepsilon) .
$$

Now, we show how to build the desired perturbed sequence form $\left(\Gamma_{\boldsymbol{S}},\left\{\ell_{i}^{\beta}\right\}_{i \in N}\right)$ associated with a given quasi-perfect equilibrium $\beta \in B$. For the ease of presentation, given $\beta \in B$, for every player $i \in N$ and sequence $s_{i} \in S_{i}$, we say that $s_{i} \in \mathcal{S}(\beta)$ if and only if $\beta_{i}(c)>0$, where $c$ is the last choice in sequence $s_{i}$, i.e., $\mathcal{S}(\beta)$ represents the support of $\beta$, which is the set of sequences whose last choice is played with positive probability in $\beta$. Then, we introduce the following definition:

Definition 7. Given a quasi-perfect equilibrium $\beta \in B$ of a game in extensive form $\Gamma$, we define its associated perturbed sequence form $\left(\Gamma_{\boldsymbol{S}},\left\{\ell_{i}^{\beta}\right\}_{i \in N}\right)$ such that, for any player $i \in N$ and sequence $s_{i} \in S_{i}$, it holds:

- if $s_{i} \in \mathcal{S}(\beta)$, then $\ell_{i}^{\beta}\left(\varepsilon, s_{i}\right)=\varepsilon^{d_{\min }^{N}\left(s_{i}^{\prime}\right)+\left|s_{i}\right|-\left|s_{i}^{\prime}\right|}$, where $s_{i}^{\prime} \in S_{i}$ is the longest sequence such that $s_{i}^{\prime} \sqsubseteq s_{i}$ (with $s_{i}^{\prime} \neq s_{i}$ ) and $s_{i}^{\prime} \notin \mathcal{S}(\beta)$, while $d_{\min }^{N}\left(s_{i}^{\prime}\right)$ is the minimum degree of $\varepsilon$ in the polynomial Poly ${ }_{i}^{N}\left(\varepsilon, s_{i}^{\prime}\right)$ appearing in the expression of $\tilde{\boldsymbol{r}}_{i}\left(\varepsilon, s_{i}^{\prime}\right)$;

- if $s_{i} \notin \mathcal{S}(\beta)$, then $\ell_{i}^{\beta}\left(\varepsilon, s_{i}\right)=\tilde{\boldsymbol{r}}_{i}\left(\varepsilon, s_{i}\right)$.

Let us remark that, given Definition 7 and how $\tilde{\boldsymbol{r}}$ is defined, it is always the case that each $\ell_{i}^{\beta}$ is well defined and satisfies the conditions of Definition 4. This is an immediate consequence of our choice for $\eta$, since, given our definition $\tilde{\boldsymbol{r}}(\varepsilon)=\boldsymbol{r}\left(\varepsilon^{\eta}\right)$, for any two distinct sequences $s_{i}, s_{i}^{\prime} \notin \mathcal{S}(\beta)$ with $s_{i}^{\prime} \sqsubseteq s_{i}$ it is always the case that $d_{\min }^{N}\left(s_{i}^{\prime}\right)-d_{\min }^{N}\left(s_{i}\right) \geq \eta$. Thus, the perturbed sequence form $\left(\Gamma_{\boldsymbol{S}},\left\{\ell_{i}^{\beta}\right\}_{i \in N}\right)$ is always well defined.

Next, we state our main result.

Theorem 3. Given a quasi-perfect equilibrium $\beta \in B$ of a game in extensive form $\Gamma$, consider its associated perturbed sequence form $\left(\Gamma_{\boldsymbol{S}},\left\{\ell_{i}^{\beta}\right\}_{i \in N}\right)$. Then, $\tilde{\boldsymbol{r}}(\varepsilon)$ defines a sequence of Nash equilibria of perturbed games $\Gamma_{\boldsymbol{S}}(\varepsilon)$ and $\beta$ is a limit point (as $\varepsilon$ goes to zero) of $\tilde{\boldsymbol{\beta}}(\varepsilon)$.

Proof. Let us consider an $\bar{\varepsilon} \in(0,1)$ such that, for all $\varepsilon \in(0, \bar{\varepsilon}), \tilde{\boldsymbol{r}}(\varepsilon)$ is a well-defined realization plan profile of $\Gamma_{\boldsymbol{S}}(\varepsilon)$ and Lemma 2 holds. Clearly, we have that $\beta$ is a limit point (as $\varepsilon$ goes to zero) of $\tilde{\boldsymbol{\beta}}(\varepsilon)$. Moreover, given Definition 7 , we have that, for every player $i \in N$, information set $u \in U_{i}$, and choice $c \in \sigma_{u}$, if $\beta_{i}(c)=0$, then $\tilde{\boldsymbol{r}}\left(\varepsilon, \sigma_{u} c\right)=\ell_{i}^{\beta}\left(\varepsilon, \sigma_{u} c\right)$, while, whenever $\beta_{i}(c)>0$, we have $\tilde{\boldsymbol{r}}\left(\varepsilon, \sigma_{u} c\right)>\ell_{i}^{\beta}\left(\varepsilon, \sigma_{u} c\right)$. Invoking Lemma 1 and Theorem 1 allows us to conclude that $\tilde{\boldsymbol{r}}(\varepsilon)$ is a Nash equilibrium of $\Gamma_{\boldsymbol{S}}(\varepsilon)$.

\subsection{Polynomial Lower Bounds are Sufficient in Two-Player Games with Nature}

A natural interesting question is whether rational functions are necessary or, instead, one can still characterize quasi-perfect equilibria by restricting the attention to a smaller family of functions. Next, we show that, in the special case of two-player games in extensive form with nature, polynomial lower bounds are indeed sufficient.

In order to show this, we prove that, in any two-player game in extensive form with nature, all the results previously presented in this section continue to hold even if we change the rational functions defining $\tilde{\boldsymbol{r}}$ so as to make them polynomial functions of $\varepsilon$. This allows us to define perturbed sequence forms $\left(\Gamma_{\boldsymbol{S}},\left\{\ell_{i}^{\beta}\right\}_{i \in N}\right)$ where the lower bounds are defined as polynomial functions. 
Next, we introduce the transformation that we need to apply to $\tilde{\boldsymbol{r}}$ in order to make it polynomial. Intuitively, we multiply all the terms depending on $\varepsilon$ that appear in the rational functions defining $\tilde{\boldsymbol{r}}$ by the same function of $\varepsilon$, which is defined in such a way that the results can be expressed as polynomials of $\varepsilon$. The following definition formally introduces our transformation.

Definition 8. Given a rational $\boldsymbol{r} \in \mathcal{R}$, letting $\Pi(\varepsilon)=\prod_{i \in N} \prod_{s_{i} \in S_{i}} \operatorname{Poly}_{i}^{D}\left(\varepsilon, s_{i}\right)$, we define a polynomial $\tilde{\boldsymbol{r}}^{p} \in \mathcal{R}$ such that:

$$
\tilde{\boldsymbol{r}}_{i}^{p}\left(\varepsilon, s_{i}\right)=a_{0}\left(s_{i}\right)+\frac{\operatorname{Poly}_{i}^{N}\left(\varepsilon^{\eta}, s_{i}\right)}{\operatorname{Poly}_{i}^{D}\left(\varepsilon^{\eta}, s_{i}\right)} \Pi\left(\varepsilon^{\eta}\right),
$$

for every player $i \in N$ and sequence $s_{i} \in S_{i}$.

Notice that $\tilde{\boldsymbol{r}}^{p}$ is obtained from $\boldsymbol{r}$ by first substituting $\varepsilon^{\eta}$ for $\varepsilon\left(\right.$ recall that $\left.\eta=\max _{i \in N, s_{i} \in S_{i}}\left|s_{i}\right|\right)$ and, then, by multiplying the terms depending on $\varepsilon$ by the product of the polynomials $\operatorname{Poly}_{i}^{D}\left(\varepsilon^{\eta}, s_{i}\right)$. In this way, the denominators in the rational functions defining $\tilde{\boldsymbol{r}}$ cancel out, obtaining polynomials.

Letting $\tilde{\boldsymbol{\beta}}^{p} \in \mathcal{P}$ be such that $\tilde{\boldsymbol{\beta}}^{p}(\varepsilon)$ has $\tilde{\boldsymbol{r}}^{p}(\varepsilon)$ as realization plan, it is a direct consequence of Definition 8 that $\boldsymbol{\beta}$ and $\tilde{\boldsymbol{\beta}}^{p}$ have the same limit points (as $\varepsilon$ goes to zero), i.e.,

$$
\lim _{\varepsilon \rightarrow 0^{+}} \boldsymbol{\beta}(\varepsilon)=\lim _{\varepsilon \rightarrow 0^{+}} \tilde{\boldsymbol{\beta}}^{p}(\varepsilon) .
$$

The following lemma (whose proof is in the Appendix) is the equivalent of Lemma 2 for $\tilde{\boldsymbol{r}}^{p}$, showing that, for any player $i \in N$ and information set $u \in U_{i}$, replacing $\boldsymbol{r}(\varepsilon)$ with $\tilde{\boldsymbol{r}}^{p}(\varepsilon)$ does not affect player $i$ 's optimal strategies in the game following $u$, provided that $\varepsilon$ is sufficiently small.

Lemma 3. Given a two-player game in sequence form $\Gamma_{\boldsymbol{S}}$ with nature ${ }^{11}$, let $\boldsymbol{r} \in \mathcal{R}$ be rational and $\tilde{\boldsymbol{r}}^{p}$ be defined as in Definition 8, then there exists $\bar{\varepsilon} \in(0,1)$ such that, for all $\varepsilon \in(0, \bar{\varepsilon})$, and for any player $i \in N$, information set $u \in U_{i}$, and choice $c \in C_{u}$, it holds:

$$
\begin{gathered}
\max _{\substack{r_{i}^{\prime} \in R_{i}: \\
r_{i}^{\prime}\left(\sigma_{u} c\right)=1}} G_{i}\left(u, \boldsymbol{r}(\varepsilon) / r_{i}^{\prime}\right)=\max _{\substack{r^{\prime} \in R_{i}: \\
r_{i}^{\prime}\left(\sigma_{u}\right)=1}} G_{i}\left(u, \boldsymbol{r}(\varepsilon) / r_{i}^{\prime}\right) \\
\max _{\substack{r_{i}^{\prime} \in R_{i}: \\
r_{i}^{\prime}\left(\sigma_{u} c\right)=1}} G_{i}\left(u, \tilde{\boldsymbol{r}}^{p}(\varepsilon) / r_{i}^{\prime}\right)=\max _{\substack{r_{i}^{\prime} \in R_{i}: \\
r_{i}^{\prime}\left(\sigma_{u}\right)=1}} G_{i}\left(u, \tilde{\boldsymbol{r}}^{p}(\varepsilon) / r_{i}^{\prime}\right) .
\end{gathered}
$$

Finally, we introduce a perturbed sequence form $\left(\Gamma_{\boldsymbol{S}},\left\{\ell_{i}^{\beta}\right\}_{i \in N}\right)$ as in Definition 7 , where we use $\tilde{\boldsymbol{r}}^{p}$ instead of $\tilde{\boldsymbol{r}}$. This results in lower bounds defined as polynomial functions of $\varepsilon$. Moreover, Theorem 3 continues to hold, provided that we employ Lemma 3 instead of Lemma 2 in its proof.

\section{Concluding Remarks}

We conclude our work with some remarks related to our characterization of quasi-perfect equilibria.

\footnotetext{
${ }^{11}$ Let us remark that Lemma 3 only holds for two-player games with nature, while it does not hold for games with more than two players. The game in Figure 2 provides a counterexample.
} 


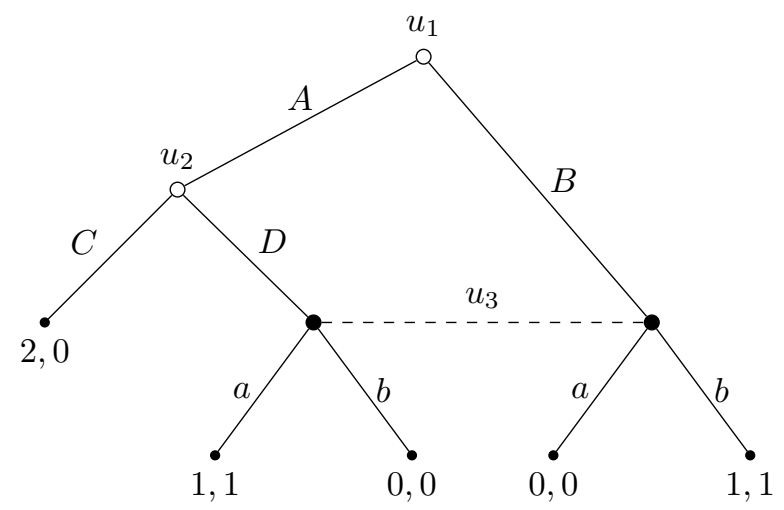

Figure 2: Example showing that perturbed sequence forms defined by rational lower bounds are necessary to characterize the quasi-perfect equilibria of games in extensive form with three or more players.

\subsection{Rational Lower Bounds are Necessary in Games with Three or More Players}

We provide an example which shows that, while polynomial lower bounds are sufficient for characterizing quasi-perfect equilibria in two-player games in extensive form with nature, rational functions are necessary in games with three or more players. This shows that our characterization of quasi-perfect equilibria is strict, since the family of functions that we prove is sufficient for the characterization cannot be further restricted.

Let us consider the game in Figure 2, where there are three players, each with a unique information set $\left(u_{1}, u_{2}\right.$, and $u_{3}$, respectively). It is easy to see that the following behavioral strategies define a quasi-perfect equilibrium of the game:

$$
\beta_{1}(A)=1, \beta_{1}(B)=0, \beta_{2}(C)=1, \beta_{2}(D)=0, \beta_{3}(a)=\frac{1}{2}, \text { and } \beta_{3}(b)=\frac{1}{2},
$$

which is obtained, e.g., for a rational $\boldsymbol{\beta} \in \mathcal{P}$ defined as follows:

$$
\boldsymbol{\beta}_{1}(\varepsilon, A)=1-\varepsilon, \boldsymbol{\beta}_{1}(\varepsilon, B)=\varepsilon, \boldsymbol{\beta}_{2}(\varepsilon, C)=\frac{1-2 \varepsilon}{1-\varepsilon}, \boldsymbol{\beta}_{2}(\varepsilon, D)=\frac{\varepsilon}{1-\varepsilon}, \text { and } \boldsymbol{\beta}_{3}(\varepsilon, a)=\boldsymbol{\beta}_{3}(\varepsilon, b)=\frac{1}{2}
$$

Moreover, it is easy to show that there is no perturbed sequence form with polynomial lower bounds that admits this quasi-perfect equilibrium as limit point of a sequence of Nash equilibria. Suppose that $\ell_{1}(\varepsilon, B)=f(\varepsilon)$ and $\ell_{2}(\varepsilon, D)=g(\varepsilon)$, where $f(\varepsilon)$ and $g(\varepsilon)$ are polynomial functions of $\varepsilon$ (while the other lower bounds can be any polynomial function of $\varepsilon$ ). Then, in a Nash equilibrium of the resulting perturbed games, it must be the case that $\boldsymbol{r}_{1}(\varepsilon, A)=1-f(\varepsilon), \boldsymbol{r}_{1}(\varepsilon, B)=f(\varepsilon)$, $\boldsymbol{r}_{2}(\varepsilon, C)=1-g(\varepsilon)$, and $\boldsymbol{r}_{2}(\varepsilon, D)=g(\varepsilon)$. Then, the third player plays both $a$ and $b$ with positive probability in a Nash equilibrium of perturbed games if and only if $(1-f(\varepsilon)) g(\varepsilon)$ (the probability of being in the left node of $u_{3}$ ) is equal to $f(\varepsilon)$ (the probability of being in the right node of $\left.u_{3}\right)$ for all $\varepsilon \in(0, \bar{\varepsilon})$, for some sufficiently small $\bar{\varepsilon} \in(0,1)$. Thus, it must be the case that $(1-f(\varepsilon)) g(\varepsilon)-f(\varepsilon)=0$ for all $\epsilon \in(0, \bar{\epsilon})$, which implies, by applying the fundamental theorem of algebra, that $(1-f(\varepsilon)) g(\varepsilon)-f(\varepsilon)=0$ for every $\varepsilon$. This implies that $(1-f(\varepsilon)) g(\varepsilon)=f(\varepsilon)$ for every $\varepsilon$, which gives $g(\varepsilon)=\frac{f(\varepsilon)}{1-f(\varepsilon)}$, contradicting the fact that $g(\varepsilon)$ is a polynomial function of $\varepsilon$.

This example allows us to conclude that, in order to capture all the quasi-perfect equilibria of a game in extensive form with three or more players, it is necessary to take into consideration perturbed sequence forms in which the lower bounds are rational functions of $\varepsilon$. 
6.2. On the Characterization of Quasi-Perfect Equilibria with Perturbed Games in Extensive Form

Our characterization of quasi-perfect equilibria as limit points of sequences of Nash equilibria of perturbed games crucially relies on the fact that trembles are defined in the sequence form. This is in contrast with most of the other Nash equilibrium refinements, which can be characterized using trembles defined in the extensive form. Nevertheless, in the following, we show that our results can also be expressed in terms of perturbed games in extensive form, though not compliant with the original definition of perturbed game provided by Selten (1975).

Let us recall that, for any game in extensive form $\Gamma$, Selten (1975) defines a perturbed game in extensive form as a pair $(\Gamma, \eta)$ where $\eta$ is a function $\eta: D \rightarrow(0,1)$ assigning a positive lower bound on the probability of playing each choice $c \in D$, such that, for any information set $u \in U$, it holds $\sum_{c \in C_{u}} \eta(c)<1$.

As discussed in Section 2.1, given a game in extensive form with perfect recall and its corresponding sequence form, we can transform behavior strategies into realization plans and vice versa, even if the transformation from realization plans to behavior strategies is not uniquely determined. However, things are slightly more complex with perturbed games.

Consider perturbed games in extensive form. Let a Selten perturbed game in extensive form $(\Gamma, \eta)$ be given. Since for any player $i \in N$, information set $u \in U_{i}$, and choice $c \in C_{u}$, it holds $\beta_{i}(c)=\frac{r_{i}\left(\sigma_{u} c\right)}{r_{i}\left(\sigma_{u}\right)} \geq \eta(c)$, we can build an associated perturbed game in sequence form in which, for any $i \in N, u \in U_{i}$, and $c \in C_{u}$, it must be the case that $r_{i}\left(\sigma_{u} c\right) \geq \eta(c) r_{i}\left(\sigma_{u}\right)$. This means that, in the associated perturbed game in sequence form, the realization plan of a sequence $\sigma_{u} c$ is subject to a lower bound which depends on the realization plan of the sequence $\sigma_{u} \cdot{ }^{12}$

On the other hand, given a perturbed sequence form $\left(\Gamma_{\boldsymbol{S}},\left\{\ell_{i}\right\}_{i \in N}\right)$, since for any $i \in N, u \in U_{i}$, and $c \in C_{u}$, it holds $r_{i}\left(\sigma_{u} c\right)=\prod_{c^{\prime} \in \sigma_{u}} \beta_{i}\left(c^{\prime}\right) \beta_{i}(c) \geq \ell_{i}\left(\varepsilon, \sigma_{u} c\right)$, we can construct an associated perturbed game in extensive form where, for any $i \in N, u \in U_{i}$, and $c \in C_{u}$, it must be the case that $\beta_{i}(c) \geq \frac{\ell_{i}\left(\varepsilon, \sigma_{u} c\right)}{\prod_{c^{\prime} \in \sigma_{u}} \beta_{i}\left(c^{\prime}\right)}$. This means that, in the associated perturbed game in extensive form, the behavior strategy of a choice $c$ is subject to a lower bound that depends on the probabilities of the choices in $\sigma_{u}$ (the sequence leading to the information set where $c$ is played). Clearly, this lower bound is not compliant with the definition of perturbed games introduced by Selten (1975), which requires that the lower bound only depends on $\varepsilon$ and not on $\beta_{i}$.

Nevertheless, it is easy to see that our results continue to hold when considering perturbed games in extensive form as defined above, thus providing a characterization of quasi-perfect equilibria as limit points of sequences of Nash equilibria of this class of perturbed games in extensive form.

\section{Acknowledgements}

We would like to thank S. Athanasoglou, K. A. Hansen, C. Pimienta, M. Serena, M. Siniscalchi, B. von Stengel, the associate editors and the three anonymous referees for helpful comments and suggestions.

\section{References}

L. Blume and W. Zame. The algebraic geometry of perfect and sequential equilibrium. Econometrica, pages 783-794, 1994.

\footnotetext{
${ }^{12}$ This result has been exploited by Farina and Gatti (2017) to show how an extensive-form perfect equilibrium can be computed by means of the sequence form.
} 
L. Blume, A. Brandenburger, and E. Dekel. Lexicographic probabilities and equilibrium refinements. Econometrica, pages 81-98, 1991.

G. Farina and N. Gatti. Extensive-form perfect equilibrium computation in two-player games. In AAAI, pages 502-508, 2017.

N. Gatti, M. Gilli, and F. Panozzo. Further results on verification problems in extensive-form games. University of Milan Bicocca Department of Economics, Management and Statistics Working Paper No. 34\%, 2016.

S. Govindan and T. Klumpp. Perfect equilibrium and lexicographic beliefs. International Journal of Game Theory, 31(2):229-243, 2003.

S. Govindan and R. Wilson. Sufficient conditions for stable equilibria. Theoretical Economics, 1 (2):167-206, 2006.

S. Govindan and R. Wilson. Axiomatic equilibrium selection for generic two-player games. Econometrica, 80(4):1639-1699, 2012.

P. Hammond. Elementary non-archimedean representations of probability for decision theory and games. In Patrick Suppes: scientific philosopher, pages 25-61. 1994.

K. A. Hansen, P. Bro Miltersen, and T. B. Sørensen. The computational complexity of trembling hand perfection and other equilibrium refinements. In International Symposium on Algorithmic Game Theory, pages 198-209. Springer, 2010.

J. Hillas, T. Kao, and A. Schiff. On a theorem of Blume and Zame. Unpublished, 2017.

E. Kohlberg and P. Reny. Independence on relative probability spaces and consistent assessments in game trees. Journal of Economic Theory, 75(2):280-313, 1997.

A. McLennan. Consistent conditional systems in noncooperative game theory. International Journal of Game Theory, 18(2):141-174, 1989.

J-F. Mertens. Two examples of strategic equilibrium. Games and Economic Behavior, 8(2):378388, 1995.

P. B. Miltersen and T. B. Sørensen. Computing a quasi-perfect equilibrium of a two-player game. Economic Theory, 42(1):175-192, 2010.

R. Myerson. Multistage games with communication. Econometrica, pages 323-358, 1986.

C. Pimienta and J. Shen. On the equivalence between (quasi-)perfect and sequential equilibria. International Journal of Game Theory, 43(2):395-402, 2014.

R. Selten. Reexamination of the perfectness concept for equilibrium points in extensive games. International journal of game theory, 4(1):25-55, 1975.

E. van Damme. A relation between perfect equilibria in extensive form games and proper equilibria in normal form games. International Journal of Game Theory, 13(1):1-13, 1984.

B. von Stengel. Efficient computation of behavior strategies. Games and Economic Behavior, 14 (2):220-246, 1996. 


\section{A. Proofs}

We provide all the proofs omitted from the main paper.

\section{A.1. Proofs Omitted From Section 3}

Before proving Theorem 1, we introduce some useful lemmas and remarks.

Lemma 4. Given a perturbed sequence form $\left(\Gamma_{\boldsymbol{S}},\left\{\ell_{i}\right\}_{i \in N}\right), r(\varepsilon)$ is a Nash equilibrium of a perturbed game in sequence form $\Gamma_{\boldsymbol{S}}(\varepsilon)$ if and only if, for every player $i \in N, \bar{r}_{i}(\varepsilon)=r_{i}(\varepsilon)-\ell_{i}(\varepsilon)$ is an optimal solution to Problem (4).

$$
\begin{array}{lll}
\max & \sum_{s \in S}\left[g_{i}(s) \prod_{j \in N \cup\{0\}, j \neq i} r_{j}\left(\varepsilon, s_{j}\right)\right] \bar{r}_{i}\left(\varepsilon, s_{i}\right) & \\
& \bar{r}_{i}(\varepsilon, \varnothing)=1-\ell_{i}(\varepsilon, \varnothing) & \text { s.t. } \\
& \sum_{c \in C_{u}} \bar{r}_{i}\left(\varepsilon, \sigma_{u} c\right)-\bar{r}_{i}\left(\varepsilon, \sigma_{u}\right)=\ell_{i}\left(\varepsilon, \sigma_{u}\right)-\sum_{c \in C_{u}} \ell_{i}\left(\varepsilon, \sigma_{u} c\right) & \forall u \in U_{i} \\
& \bar{r}_{i}\left(\varepsilon, s_{i}\right) \geq 0 & \forall s_{i} \in S_{i} .
\end{array}
$$

Proof. In a Nash equilibrium every player must play a best response to the opponents' strategies. Let us fix a player $i \in N$. Consider the best-response problem of player $i$ against $r(\varepsilon)$ in $\Gamma_{\boldsymbol{S}}(\varepsilon)$, which reads as follows:

$$
\begin{array}{lll}
\max & \sum_{s \in S} g_{i}(s) \prod_{j \in N \cup\{0\}} r_{j}\left(\varepsilon, s_{j}\right) & \\
& r_{i}(\varepsilon, \varnothing)=1 & \\
& r_{i}\left(\varepsilon, \sigma_{u}\right)=\sum_{c \in C_{u}} r_{i}\left(\varepsilon, \sigma_{u} c\right) & \forall u \in U_{i} \\
& r_{i}\left(\varepsilon, s_{i}\right) \geq \ell_{i}\left(\varepsilon, s_{i}\right) & \forall s_{i} \in S_{i} .
\end{array}
$$

Then, by introducing variables $\bar{r}_{i}\left(\varepsilon, s_{i}\right)=r_{i}\left(s_{i}\right)-\ell_{i}\left(\varepsilon, s_{i}\right)$ for $s_{i} \in S_{i}$, the problem can be rewritten as follows:

$$
\begin{array}{rlr}
\max & \sum_{s \in S}\left[g_{i}(s) \prod_{j \in N \cup\{0\}, j \neq i} r_{j}\left(\varepsilon, s_{j}\right)\right] \bar{r}_{i}\left(\varepsilon, s_{i}\right)+\sum_{s \in S}\left[g_{i}(s) \prod_{j \in N \cup\{0\}, j \neq i} r_{j}\left(\varepsilon, s_{j}\right)\right] \ell_{i}\left(\varepsilon, s_{i}\right) & \\
& \bar{r}_{i}(\varepsilon, \varnothing)+\ell_{i}(\varepsilon, \varnothing)=1 \\
& \bar{r}_{i}\left(\varepsilon, \sigma_{u}\right)+\ell_{i}\left(\varepsilon, \sigma_{u}\right)=\sum_{c \in C_{u}} \bar{r}_{i}\left(\varepsilon, \sigma_{u} c\right)+\sum_{c \in C_{u}} \ell_{i}\left(\varepsilon, \sigma_{u} c\right) & \forall u \in U_{i} \\
& \bar{r}_{i}\left(\varepsilon, s_{i}\right) \geq 0 & \forall s_{i} \in S_{i} .
\end{array}
$$

which, by rearranging the terms in the constraints, is equivalent to Problem (4) since the second term in the objective function is a constant.

Problem (4) is a linear program, as all players' realization plans are fixed, except for that of player $i$. In the following lemma, we introduce the dual of Problem (4). 
Lemma 5. Given a perturbed sequence form $\left(\Gamma_{\boldsymbol{S}},\left\{\ell_{i}\right\}_{i \in N}\right)$, for every realization plan profile $r(\varepsilon)$ of $\Gamma_{\boldsymbol{S}}(\varepsilon)$ and player $i \in N$, Problem (5) is the dual of Problem (4), where the variables $v(u)$, for $u \in U_{i}$, denote the dual variables associated with Constraints (4c).

$$
\begin{array}{ll}
\min & \sum_{u \in U_{i}}\left(\ell_{i}\left(\varepsilon, \sigma_{u}\right)-\sum_{c \in C_{u}} \ell_{i}\left(\varepsilon, \sigma_{u} c\right)\right) v(u) \\
v(u) \geq \sum_{\substack{s \in S: \\
s_{i}=\sigma_{u} c}}\left[g_{i}(s) \prod_{j \in N \cup\{0\}, j \neq i} r_{j}\left(\varepsilon, s_{j}\right)\right]+\sum_{\substack{u^{\prime} \in U_{i}: \\
\sigma_{u}=\sigma_{u} c}} v\left(u^{\prime}\right) \quad \forall u \in U_{i}, \forall c \in C_{u} .
\end{array}
$$

Proof. The dual of Problem (4) reads as follows:

$$
\begin{array}{ll}
\min & \left(1-\ell_{i}(\varepsilon, \varnothing)\right) v(\varnothing)+\sum_{u \in U_{i}}\left(\ell_{i}\left(\varepsilon, \sigma_{u}\right)-\sum_{c \in C_{u}} \ell_{i}\left(\varepsilon, \sigma_{u} c\right)\right) v(u) \\
v(\varnothing) \geq \sum_{\substack{s \in S: \\
s_{i}=\varnothing}}\left[g_{i}(s) \prod_{j \in N \cup\{0\}, j \neq i} r_{j}\left(\varepsilon, s_{j}\right)\right]+\sum_{\substack{u^{\prime} \in U_{i}: \\
\sigma_{u^{\prime}}=\varnothing}} v\left(u^{\prime}\right) \\
v(u) \geq \sum_{\substack{s \in S: \\
s_{i}=\sigma_{u} c}}\left[g_{i}(s) \prod_{j \in N \cup\{0\}, j \neq i} r_{j}\left(\varepsilon, s_{j}\right)\right]+\sum_{\substack{u^{\prime} \in U_{i}: \\
\sigma_{u^{\prime}}=\sigma_{u} c}} v\left(u^{\prime}\right)
\end{array} \quad \forall u \in U_{i}, \forall c \in C_{u},
$$

where $v(\varnothing)$ and $v(u)$, for $u \in U_{i}$, denote the dual variables associated with Constraints (4b)-(4c). Since $\ell_{i}(\varepsilon, \varnothing)=1$ by Definition 4 , we can remove variable $v(\varnothing)$ and obtain the result.

The optimal solutions to Problem (5) enjoy some particular properties, which are stated in the following lemmas. The first lemma says that, in an optimal solution, each variable $v(u)$ is set to the value of player $i$ 's expected utility for an optimal strategy in the game following information set $u$, and this holds for any $u \in U_{i}$ because of perturbations.

Lemma 6. Given a perturbed sequence form $\left(\Gamma_{\boldsymbol{S}},\left\{\ell_{i}\right\}_{i \in N}\right)$, for every realization plan profile $r(\varepsilon)$ of $\Gamma_{\boldsymbol{S}}(\varepsilon)$ and player $i \in N$, the values $v^{*}(u)$, for $u \in U_{i}$, constitute an optimal solution to Problem (5) if and only if, for every $u \in U_{i}$, it holds:

$$
v^{*}(u)=\max _{\substack{r_{i}^{\prime} \in R_{i}: \\ r_{i}^{\prime}\left(\sigma_{u}\right)=1}} G_{i}\left(u, r(\varepsilon) / r_{i}^{\prime}\right) .
$$

Proof. Let us consider Problem (5), First, observe that the objective function coefficient for the variable $v(u)$ associated with $u \in U_{i}$ is equal to $\ell_{i}\left(\varepsilon, \sigma_{u}\right)-\sum_{c \in C_{u}} \ell_{i}\left(\varepsilon, \sigma_{u} c\right)$, which is positive by Definition 4. Thus, in an optimal solution $v^{*}(u)$ (for $u \in U_{i}$ ), each variable $v(u)$ is set to its minimum value given Constraints (5b).

$\left(\Longleftarrow\right.$ ) Suppose $v^{*}(u)$ (for $u \in U_{i}$ ) is an optimal solution to Problem (5). We prove Equation (6) using a simple inductive argument. The base case of the induction is when there is no information set $u^{\prime} \in U_{i}$ with $u \prec u^{\prime}$. For every choice $c \in C_{u}$, it holds

$$
v(u) \geq \sum_{s \in S: s_{i}=\sigma_{u} c}\left[g_{i}(s) \prod_{j \in N \cup\{0\}, j \neq i} r_{j}\left(\varepsilon, s_{j}\right)\right],
$$


which, using the fact that $v^{*}(u)$ must be set to its minimum possible value given the constraints, implies the following:

$$
v^{*}(u)=\max _{c \in C_{u}} \sum_{\substack{s \in S: \\ s_{i}=\sigma_{u} c}}\left[g_{i}(s) \prod_{j \in N \cup\{0\}, j \neq i} r_{j}\left(\varepsilon, s_{j}\right)\right]=\max _{\substack{r_{i}^{\prime} \in R_{i}: \\ r_{i}^{\prime}\left(\sigma_{u}\right)=1}} G_{i}\left(u, r(\varepsilon) / r_{i}^{\prime}\right),
$$

where the last equality holds since there is no $u^{\prime} \in U_{i}$ such that $u \prec u^{\prime}$ and $\sum_{c \in C_{u}} r_{i}^{\prime}\left(\sigma_{u} c\right)=r_{i}^{\prime}\left(\sigma_{u}\right)=1$, for the definition of realization plan. As for the inductive step, let us consider an information set $u \in U_{i}$ and assume, by induction, that Equation (6) holds for every information set $u^{\prime} \in U_{i}$ with $u \prec u^{\prime}$. We can write:

$$
\begin{aligned}
v^{*}(u) & =\max _{c \in C_{u}}\left\{\sum_{\substack{s \in S: \\
s_{i}=\sigma_{u} c}}\left[g_{i}(s) \prod_{j \in N \cup\{0\}, j \neq i} r_{j}\left(\varepsilon, s_{j}\right)\right]+\sum_{\substack{u^{\prime} \in U_{i}: \\
\sigma_{u^{\prime}}=\sigma_{u} c}} v^{*}\left(u^{\prime}\right)\right\}= \\
& =\max _{c \in C_{u}}\left\{\sum_{\substack{s \in S: \\
s_{i}=\sigma_{u} c}}\left[g_{i}(s) \prod_{j \in N \cup\{0\}, j \neq i} r_{j}\left(\varepsilon, s_{j}\right)\right]+\sum_{\substack{u^{\prime} \in U_{i}: \\
\sigma_{u^{\prime}}=\sigma_{u} c}} \max _{\substack{r_{i}^{\prime} \in R_{i}: \\
r_{i}^{\prime}\left(\sigma_{u^{\prime}}\right)=1}} G_{i}\left(u, r(\varepsilon) / r_{i}^{\prime}\right)\right\}= \\
& =\max _{\substack{r_{i}^{\prime} \in R_{i}: \\
r_{i}^{\prime}\left(\sigma_{u}\right)=1}} G_{i}\left(u, r(\varepsilon) / r_{i}^{\prime}\right),
\end{aligned}
$$

where the first equality directly follows from the optimality of $v^{*}\left(u^{\prime}\right)$, the second one from the inductive hypothesis, while the last equality holds since we have $\sum_{c \in C_{u}} r_{i}^{\prime}\left(\sigma_{u} c\right)=r_{i}^{\prime}\left(\sigma_{u}\right)=1$.

$(\Longrightarrow)$ By contradiction, suppose that Equation (6) holds for every information set $u \in U_{u}$ and $v^{*}(u)$ (for $u \in U_{i}$ ) is not an optimal solution to Problem (5). Thus, there must be an information set $u \in U_{i}$ such that Equation (6) holds for all $u^{\prime} \in U_{i}$ with $u \prec u^{\prime}$ and $v^{*}(u)$ satisfies all the Constraints (5b) with strict inequality (since it is not optimal). Therefore,

$$
\begin{aligned}
v^{*}(u) & >\max _{c \in C_{u}}\left\{\sum_{\substack{s \in S: \\
s_{i}=\sigma_{u} c}}\left[g_{i}(s) \prod_{j \in N \cup\{0\}, j \neq i} r_{j}\left(\varepsilon, s_{j}\right)\right]+\sum_{\substack{u^{\prime} \in U_{i}: \\
\sigma_{u^{\prime}}=\sigma_{u} c}} v^{*}\left(u^{\prime}\right)\right\}= \\
& =\max _{\substack{r_{i}^{\prime} \in R_{i}: \\
r_{i}^{\prime}\left(\sigma_{u}\right)=1}} G_{i}\left(u, r(\varepsilon) / r_{i}^{\prime}\right),
\end{aligned}
$$

which contradicts Equation (6) for the information set $u$.

The following lemma says that, if an optimal solution to Problem (5) satisfies Constraint (5b) with equality, for an information set $u$ and a choice $c \in C_{u}$, then playing choice $c$ at $u$ is part of an optimal strategy in the game following $u$, and this holds for any $u \in U_{i}$ because of perturbations.

Lemma 7. Given a perturbed sequence form $\left(\Gamma_{\boldsymbol{S}},\left\{\ell_{i}\right\}_{i \in N}\right)$, for every realization plan profile $r(\varepsilon)$ of $\Gamma_{\boldsymbol{S}}(\varepsilon)$, player $i \in N$, information set $u \in U_{i}$, and choice $c \in C_{u}$, Constraint (5b) holds with equality in an optimal solution to Problem (5) if and only if:

$$
\max _{\substack{r_{i}^{\prime} \in R_{i}: \\ r_{i}^{\prime}\left(\sigma_{u} c\right)=1}} G_{i}\left(u, r(\varepsilon) / r_{i}^{\prime}\right)=\max _{\substack{r_{i}^{\prime} \in R_{i}: \\ r_{i}^{\prime}\left(\sigma_{u}\right)=1}} G_{i}\left(u, r(\varepsilon) / r_{i}^{\prime}\right) .
$$


Proof. In the following, let $v^{*}(u)$ (for $u \in U_{i}$ ) be optimal for Problem (5).

$(\Longrightarrow)$ Because of Lemma 6 and Equation $(7)$, we can write:

$$
\begin{aligned}
v^{*}(u) & =\max _{\substack{r_{i}^{\prime} \in R_{i}: \\
r_{i}^{\prime}\left(\sigma_{u}\right)=1}} G_{i}\left(u, r(\varepsilon) / r_{i}^{\prime}\right)=\max _{\substack{r_{i}^{\prime} \in R_{i} \\
r_{i}^{\prime}\left(\sigma_{u} c\right)=1}} G_{i}\left(u, r(\varepsilon) / r_{i}^{\prime}\right)= \\
& =\sum_{\substack{s \in S: \\
s_{i}=\sigma_{u} c}}\left[g_{i}(s) \prod_{j \in N \cup\{0\}, j \neq i} r_{j}\left(\varepsilon, s_{j}\right)\right]+\sum_{\substack{u^{\prime} \in U_{i}: \\
\sigma_{u^{\prime}}=\sigma_{u} c}} v^{*}\left(u^{\prime}\right) .
\end{aligned}
$$

$(\Longleftarrow)$ Using Lemma 6 we can write:

$$
\begin{aligned}
v^{*}(u) & =\sum_{\substack{s \in S: \\
s_{i}=\sigma_{u} c}}\left[g_{i}(s) \prod_{j \in N \cup\{0\}, j \neq i} r_{j}\left(\varepsilon, s_{j}\right)\right]+\sum_{\substack{u^{\prime} \in U_{i}: \\
\sigma_{u^{\prime}}=\sigma_{u} c}} v^{*}\left(u^{\prime}\right)= \\
& =\max _{\substack{r_{i}^{\prime} \in R_{i} \\
r_{i}^{\prime}\left(\sigma_{u} c\right)=1}} G_{i}\left(u, r(\varepsilon) / r_{i}^{\prime}\right)=\max _{\substack{r_{i}^{\prime} \in R_{i}: \\
r_{i}^{\prime}\left(\sigma_{u}\right)=1}} G_{i}\left(u, r(\varepsilon) / r_{i}^{\prime}\right) .
\end{aligned}
$$

This concludes the proof.

We are now ready to prove Theorem 1.

Proof of Theorem 1. By Lemma 4, $r(\varepsilon)$ is a Nash equilibrium of $\Gamma_{\boldsymbol{S}}(\varepsilon)$ if and only if, for every player $i \in N, \bar{r}_{i}(\varepsilon)=r_{i}(\varepsilon)-\ell_{i}(\varepsilon)$ is an optimal solution to Problem (4). The result is a direct application of the complementarity slackness theorem in linear programming (using the conditions connecting the primal variables with the slack variables of the corresponding dual constraints). Let us fix $i \in N, u \in U_{i}$, and $c \in C_{u}$. By applying the theorem to Problems (4) and (5) we can conclude that $\bar{r}_{i}(\varepsilon)$ and $v^{*}(u)$ (for $u \in U_{i}$ ) are optimal if and only if, whenever $\bar{r}_{i}\left(\varepsilon, \sigma_{u} c\right)>0$ (i.e., equivalently, $r_{i}\left(\varepsilon, \sigma_{u} c\right)>\ell_{i}\left(\varepsilon, \sigma_{u} c\right)$ ), then Constraint (5b) for information set $u$ and choice $c$ holds with equality, which, using Lemma 7, implies Equation (7). Since the two conditions hold for every player $i \in N$, information set $u \in U_{i}$, and choice $c \in C_{u}$, the proof is complete.

\section{A.2. Proofs Omitted From Section 4}

Proof of Lemma 1. First, let us notice that the following relation holds:

$$
\begin{aligned}
\max _{\substack{r_{i}^{\prime} \in R_{i}: \\
r_{i}^{\prime}\left(\sigma_{u} c\right)=1}} G_{i}\left(u, r / r_{i}^{\prime}\right) & =\max _{\substack{r_{i}^{\prime} \in R_{i}: \\
r_{i}^{\prime}\left(\sigma_{u}\right)=1}} G_{i}\left(u, r / r_{i}^{\prime}\right) \Longleftrightarrow \\
\max _{\beta_{i}^{\prime} \in B_{i}: \beta_{i}^{\prime}(c)=1} H_{i}\left(\beta /{ }_{u} \beta_{i}^{\prime} \mid u\right) & =\max _{\beta_{i}^{\prime} \in B_{i}} H_{i}\left(\beta /{ }_{u} \beta_{i}^{\prime} \mid u\right) .
\end{aligned}
$$

In order to see this, for $z \in Z(u)$ (i.e., $z$ is a terminal node reachable from $u$ ) and $\beta_{i}^{\prime} \in B_{i}$, let $H_{i}\left(z, \beta, \beta_{i}^{\prime}\right)$ be such that:

$$
H_{i}\left(z, \beta, \beta_{i}^{\prime}\right)=h_{i}(z)\left(\prod_{j \in N \cup\{0\}, j \neq i} \prod_{c \in p(z) \cap D_{j}} \beta_{j}(c)\right)\left(\prod_{c \in p(z) \cap D_{i}: c \notin \sigma_{u}} \beta_{i}^{\prime}(c)\right),
$$


where we recall that $p(z)$ represents the set of choices on the path from the root of the game to $z$ (see Table 1). Since $r$ is the realization plan of $\beta$ and $r_{i}^{\prime}\left(\sigma_{u}\right)=1$, the first line of Equation (8) is the same as:

$$
\max _{\beta_{i}^{\prime} \in B_{i}: \beta_{i}^{\prime}(c)=1} \sum_{z \in Z(u)} H_{i}\left(z, \beta, \beta_{i}^{\prime}\right)=\max _{\beta_{i}^{\prime} \in B_{i}} \sum_{z \in Z(u)} H_{i}\left(z, \beta, \beta_{i}^{\prime}\right) .
$$

Then, by dividing both sides of the equality by $\sum_{x \in u} \prod_{j \in N \cup\{0\}, j \neq i} \prod_{c \in p(x) \cap D_{j}} \beta_{i}(c)$ and using the definition of $H_{i}(\beta \mid u)$, i.e.,

$$
H_{i}(\beta \mid u)=\frac{\sum_{z \in Z(u)}\left[h_{i}(z) \prod_{j \in N \cup\{0\}} \prod_{c \in p(z) \cap D_{j}} \beta_{j}(c)\right]}{\sum_{x \in u} \prod_{j \in N \cup\{0\}} \prod_{c \in p(x) \cap D_{j}} \beta_{j}(c)}=\frac{\sum_{z \in Z(u)}\left[h_{i}(z) \prod_{j \in N \cup\{0\}} \prod_{c \in p(z) \cap D_{j}: c \notin \sigma_{u}} \beta_{j}(c)\right]}{\sum_{x \in u} \prod_{j \in N \cup\{0\}, j \neq i} \prod_{c \in p(x) \cap D_{j}} \beta_{j}(c)},
$$

we get the second line of Equation (8); where by $c \notin \sigma_{u}$ we mean that choice $c$ is not contained in the sequence $\sigma_{u}$. Clearly, we can prove the other direction of Equation (8) following the same steps in the reverse order (and multiplying instead of dividing).

$(\Longrightarrow)$ Suppose that the condition holds for every choice $c \in C_{u}$. Clearly,

$$
\max _{\beta_{i}^{\prime} \in B_{i}: \beta_{i}=\beta_{u}^{\prime}} H_{i}\left(\beta /{ }_{u} \beta_{i}^{\prime} \mid u\right)=\sum_{c \in C_{u}} \beta_{i}(c) \max _{\beta_{i}^{\prime} \in B_{i}: \beta_{i}^{\prime}(c)=1} H_{i}\left(\beta /{ }_{u} \beta_{i}^{\prime} \mid u\right)
$$

and, since $\beta_{i}(c)>0$ only if $\max _{\substack{r_{i}^{\prime} \in R_{i} \\ r_{i}^{\prime}\left(\sigma_{u} c\right)=1}} G_{i}\left(u, r / r_{i}^{\prime}\right)=\max _{\substack{r_{i}^{\prime} \in R_{i}: \\ r_{i}^{\prime}\left(\sigma_{u}\right)=1}} G_{i}\left(u, r / r_{i}^{\prime}\right)$, a direct application of Equation (8) proves the result.

$(\Longleftarrow)$ As for the other direction, let $\beta_{i} \in \mathcal{B R}_{u}(\beta)$ be given. Thus, it is the case that the following holds: $\max _{\beta_{i}^{\prime} \in B_{i}: \beta_{i}=\beta_{i}^{\prime}} H_{i}\left(\beta /{ }_{u} \beta_{i}^{\prime} \mid u\right)=\max _{\beta_{i}^{\prime} \in B_{i}} H_{i}\left(\beta /{ }_{u} \beta_{i}^{\prime} \mid u\right)$. By contradiction, suppose that $\max _{\substack{r_{i}^{\prime} \in R_{i} \\ r_{i}^{\prime}\left(\sigma_{u} c\right)=1}} G_{i}\left(u, r / r_{i}^{\prime}\right)<\max _{\substack{r_{i}^{\prime} \in R_{i}: \\ r_{i}^{\prime}\left(\sigma_{u}\right)=1}} G_{i}\left(u, r / r_{i}^{\prime}\right)$ for some choice $c \in C_{u}$ such that $\beta_{i}(c)>0$. Because of Equation (8), we would have

$$
\max _{\beta_{i}^{\prime} \in B_{i}: \beta_{i}^{\prime}(c)=1} H_{i}\left(\beta /{ }_{u} \beta_{i}^{\prime} \mid u\right)<\max _{\beta_{i}^{\prime} \in B_{i}} H_{i}\left(\beta /{ }_{u} \beta_{i}^{\prime} \mid u\right)
$$

which implies the following:

$$
\begin{aligned}
\max _{\beta_{i}^{\prime} \in B_{i}: \beta_{i}=\beta_{i}^{\prime}} H_{i}\left(\beta /{ }_{u} \beta_{i}^{\prime} \mid u\right) & =\sum_{c \in C_{u}} \beta_{i}(c) \max _{\beta_{i}^{\prime} \in B_{i}: \beta_{i}^{\prime}(c)=1} H_{i}\left(\beta /{ }_{u} \beta_{i}^{\prime} \mid u\right) \\
& <\max _{\beta_{i}^{\prime} \in B_{i}} H_{i}\left(\beta /{ }_{u} \beta_{i}^{\prime} \mid u\right),
\end{aligned}
$$

contradicting $\beta_{i} \in \mathcal{B R}_{u}(\beta)$.

\section{A.3. Proofs Omitted From Section 5}

Proof of Lemma 2. Let us fix a player $i \in N$, an information set $u \in U_{i}$, and a choice $c \in C_{u}$. First, notice that $G_{i}\left(u, \boldsymbol{r}(\varepsilon) / r_{i}^{\prime}\right)$ can be expressed as a linear combination (with coefficients depending 
on $\varepsilon$ ) of the variables $r_{i}^{\prime}\left(s_{i}\right)$, where the sequences $s_{i}$ are those containing a sequence $\sigma_{u} c^{\prime}$ for some $c^{\prime} \in C_{u}$. Formally,

$$
G_{i}\left(u, \boldsymbol{r}(\varepsilon) / r_{i}^{\prime}\right)=\sum_{c^{\prime} \in C_{u}} \sum_{s_{i} \in S_{i}: \sigma_{u} c^{\prime} \sqsubseteq s_{i}} r_{i}^{\prime}\left(s_{i}\right) \gamma_{i}\left(\varepsilon, s_{i}\right),
$$

where, given that $\boldsymbol{r}$ is rational, each $\gamma_{i}\left(\varepsilon, s_{i}\right)$ is a rational function of $\varepsilon$. Given that the terms $\gamma_{i}\left(\varepsilon, s_{i}\right)$ are expressed as rational functions of $\varepsilon$, there must exist a sufficiently small $\bar{\varepsilon} \in(0,1)$ such that, for all $\varepsilon \in(0, \bar{\varepsilon})$, the relative ordering of the terms $\gamma_{i}\left(\varepsilon, s_{i}\right)$ is the same. Formally, there exists an ordering $s_{i}^{(1)}, s_{i}^{(2)}, \ldots, s_{i}^{(k)}$ of the sequences $s_{i} \in S_{i}: \sigma_{u} c^{\prime} \sqsubseteq s_{i}$ for some $c^{\prime} \in C_{u}$ such that, for all $\varepsilon \in(0, \bar{\varepsilon})$, it is the case that:

$$
\gamma_{i}\left(\varepsilon, s_{i}^{(1)}\right) \geq \gamma_{i}\left(\varepsilon, s_{i}^{(2)}\right) \geq \ldots \geq \gamma_{i}\left(\varepsilon, s_{i}^{(k)}\right)
$$

where $k$ is the number of such sequences. Let us remark that, if for two sequences $s_{i}^{(j)}$ and $s_{i}^{(j+1)}$ the above relation holds with equality, i.e., $\gamma_{i}\left(\varepsilon, s_{i}^{(j)}\right)=\gamma_{i}\left(\varepsilon, s_{i}^{(j+1)}\right)$, then $\gamma_{i}\left(\varepsilon, s_{i}^{(j)}\right)$ and $\gamma_{i}\left(\varepsilon, s_{i}^{(j+1)}\right)$ are defined by the same rational function of $\varepsilon$. Now, let us define $\tilde{\gamma}_{i}\left(\varepsilon, s_{i}\right)=\gamma_{i}\left(\varepsilon^{\eta}, s_{i}\right)$ for all sequences $s_{i} \in S_{i}: \sigma_{u} c^{\prime} \sqsubseteq s_{i}$ for some $c^{\prime} \in C_{u}$. Given that they are rational functions of $\varepsilon$, there must exist a (different, in general) sufficiently small $\bar{\varepsilon} \in(0,1)$ such that, for all $\varepsilon \in(0, \bar{\varepsilon})$, the ordering of the sequences is preserved, i.e.,

$$
\tilde{\gamma}_{i}\left(\varepsilon, s_{i}^{(1)}\right) \geq \tilde{\gamma}_{i}\left(\varepsilon, s_{i}^{(2)}\right) \geq \ldots \geq \tilde{\gamma}_{i}\left(\varepsilon, s_{i}^{(k)}\right) .
$$

Given that we can write

$$
G_{i}\left(u, \tilde{\boldsymbol{r}}(\varepsilon) / r_{i}^{\prime}\right)=\sum_{c^{\prime} \in C_{u}} \sum_{s_{i} \in S_{i}: \sigma_{u} c^{\prime} \sqsubseteq s_{i}} r_{i}^{\prime}\left(s_{i}\right) \tilde{\gamma}_{i}\left(\varepsilon, s_{i}\right),
$$

by using the fact that $G_{i}\left(u, \boldsymbol{r}(\varepsilon) / r_{i}^{\prime}\right)$ and $G_{i}\left(u, \tilde{\boldsymbol{r}}(\varepsilon) / r_{i}^{\prime}\right)$ are linear functions of $r_{i}^{\prime}$, we can conclude that, for $\varepsilon \in(0, \bar{\varepsilon})$, if the two max expressions on the left-hand side of the condition stated in the lemma are the same, then the same holds for the two appearing in the right-hand side, and vice versa. Since these arguments hold for every $i \in N, u \in U_{i}$, and $c \in C_{u}$, it is sufficient to take a sufficiently small $\bar{\varepsilon} \in(0,1)$ to conclude the proof.

\section{A.4. Proofs Omitted From Section 6}

Proof of Lemma 3. The proof follows the line of that of Lemma 2. Since the game has only two players, it holds $N=\{1,2\}$, and, thus, for the ease of presentation, given a player $i \in N$, we let $j$ be her opponent. Once fixed $i \in N, u \in U_{i}$, and $c \in C_{u}$, the proof follows the same steps as that of Lemma 2, where, in this case, we define $\tilde{\gamma}_{i}\left(\varepsilon, s_{i}\right)=\gamma_{i}\left(\varepsilon^{\eta}, s_{i}\right) \Pi(\varepsilon)$. Remember that $\Pi(\varepsilon)=\prod_{i \in N} \prod_{s_{i}^{\prime} \in S_{i}} \operatorname{Poly}_{i}^{D}\left(\varepsilon, s_{i}^{\prime}\right)$. Notice that, in a two-player game with nature, $\gamma_{i}\left(\varepsilon, s_{i}\right)$ is the sum of rational functions of $\varepsilon$ coming from the realization plan $r_{j}(\varepsilon)$, possibly multiplied by the probabilities of chance moves (not depending on $\varepsilon$ ). Formally;

$$
\gamma_{i}\left(\varepsilon, s_{i}\right)=\sum_{s_{j} \in S_{j}} \sum_{s_{0} \in S_{0}} g_{i}\left(s_{0}, s_{i}, s_{j}\right) r_{0}\left(s_{0}\right) r_{j}\left(\varepsilon, s_{j}\right)
$$

where we write $r_{0}\left(s_{0}\right)$ in place of $r_{0}\left(\varepsilon, s_{0}\right)$ as it does not depend on $\varepsilon$. As a result, we can write:

$$
G_{i}\left(u, \tilde{\boldsymbol{r}}^{p}(\varepsilon) / r_{i}^{\prime}\right)=\sum_{c^{\prime} \in C_{u}} \sum_{s_{i} \in S_{i}: \sigma_{u} c^{\prime} \sqsubseteq s_{i}} r_{i}^{\prime}\left(s_{i}\right) \tilde{\gamma}_{i}\left(\varepsilon, s_{i}\right)
$$


since all the terms depending on $\varepsilon$ are multiplied by $\Pi(\varepsilon)$ only one time in $\tilde{\boldsymbol{r}}^{p}(\varepsilon)$. Notice that this does not hold with more than two players, as in $\gamma_{i}\left(\varepsilon, s_{i}\right)$ probabilities defined by the realization plans of different players may be multiplied. In conclusion, all the steps in the proof of Lemma 2 continue to hold since $\Pi(\varepsilon)$ goes to one as $\varepsilon$ goes to zero. 